\title{
Diverse Transformers (Trf) protein family in the sea urchin Paracentrotus lividus act through collaboration between cellular and humoral immune effector arms
}

\author{
Iryna Yakovenko ${ }^{1}$, Asaf Donnyo ${ }^{1}$, Or Ioscovich ${ }^{1}$, Benyamin Rosental $^{2}$, Matan Oren $^{1 *}$
}

Affiliations:

${ }^{1}$ Department of Molecular Biology, Ariel University, Science Park, Ariel 40700, Israel

${ }^{2}$ The Shraga Segal Department of Microbiology, Immunology and Genetics, Regenerative Medicine, and Stem Cell Research Center, Ben-Gurion University of the Negev, Beer Sheva 84105, Israel

* Correspondence to: matanor@ariel.ac.il

\begin{abstract}
Sea urchins are long-living invertebrates with a complex immune system which includes extended families of immune receptors. A central immune gene family in the sea urchins encodes for the Transformer (Trf) proteins. The Trf family was so far studied mainly in the purple sea urchin Strongylocentrotus purpuratus. In this study, we explored this protein family in the Mediterranean Sea urchin Paracentrotus lividus. The PlTrf genes and predicted proteins were found to be highly diverse and showed a typical Trf size range and structure. We found that P. lividus coelomocytes and hemolymph contain different PITrf protein repertoires with a shared subset which specifically bind $E$. coli bacteria. Using FACS, we identified five different $P$. lividus coelomocyte sub-populations with cell surface Trf protein expression. The relative abundance of the Trf-positive cells sharply increased following immune challenge with $E$. coli bacteria, but not following challenge with LPS or sea urchin pathogen $V$. penaeicida. Finally, we demonstrated that the phagocytosis of E. coli bacteria by $P$. lividus phagocytes is mediated through the hemolymph and is inhibited by blocking Trf activity with anti-Trf antibodies. Together, our results suggest collaboration between cellular and humoral Trf-mediated effector arms in the $P$. lividus specific immune response to pathogens.
\end{abstract}

Keywords: Paracentrotus lividus, Transformers, Trf, 185/333, invertebrate immunity, phagocytosis, model organism

\section{Introduction}

Sea urchins are long-living invertebrates that are constantly being exposed in nature to the marine environment and its pathogens through direct contact with marine substrates, seawater, and consumed food. For protection, they have evolved a very sophisticated and robust immune system with multiple effector arms and a wide genome representation of immune genes [1]. The echinoid immune gene repertoire includes several extended immune gene families that encode for receptors and hemolymph proteins that participate in either cellular responses (e.g., Toll-like receptors, NOD-like receptors, scavenger receptor) or humoral responses (e.g., C-type lectins, complement such as factor B) [2, 3]. In spite of the absence of vertebrate-like adaptive immunity, the large number of the germline-encoded immune proteins in the sea urchins suggest that they may specifically recognize and act against a variety of pathogen-associated molecular patterns (PAMPs). One of the central immune gene families in sea urchin's immune response encodes for the Transformer (Trf) proteins.

The Trf protein family (formerly termed 185/333) was first characterized in the California purple sea urchin Strongylocentrotus Purpuratus [4]. Since then, more than 30 studies about the family in this species were published and only one study in a different sea urchin species - Heliocidaris erythrogramma [5]. To date, no $\operatorname{Trf}$ genes or transcript sequences have been identified outside of the echinoid lineage, suggesting that Trf-based immunity is unique to sea urchins. In S. purpuratus, a robust Trf-mediated response was documented upon challenge with bacteria including Escherichia coli and Vibrio diazotrophicus $[6,7]$ and with PAMPs including lipopolysaccharide (LPS), $\beta-1,3$-glucan and double-stranded RNA [8]. The response was characterized by a significant increase in SpTrf transcript 
and protein quantities [4, 6, 8-10] as well as in increasing Trf-expressing cell ratios [11-13]. The $\operatorname{SpTrf}$ proteins are capable to selectively bind to different antigens [14], augment phagocytosis and retard bacterial growth [15]. It was also shown that a recombinant SpTrf protein is transforming its shape upon binding to antigens. Consequently, the family name was recently changed from 185/333 to Transformers [16].

The $\operatorname{SpTrf}$ genes are 1.2 - $2 \mathrm{~kb}$ long and consist of two exons; a short leader and a second exon which encodes for the functional protein [4]. The structure of the second exon is made of a mosaic of blocks of conserved sequences, termed elements, that are either present or absent in different members of the family $[4,9,17]$. The defined combinations of the gene elements of a second exon are the primary source of diversity in gene sequence and size. However, additional diversification processes occur downstream, post-transcription [18] and post-translation, resulting in a diversity of up to 260 different SpTrf proteins per individual as was deduced from 2 DE Western blot analysis $[6,10]$. Fifteen $\operatorname{Sp} \operatorname{Tr} f$ genes were identified within two to three gene clusters in the genome of a single sea urchin [19]. However, the $\operatorname{SpTrf}$ loci might be highly unstable due to gene clustering and tandem sequence repeats [20] and the SpTrf gene repertoire show high variability within the sea urchin population [19]. Furthermore, it was shown that $\operatorname{SpTrf}$ genes may be subjected to regulated genomic diversification processes leading to gene deletions, duplications and single point mutations (SNPs) in single cells [12] and that each cell may express a unique single $\operatorname{SpTrf}$ message [7].

The Trf proteins are intrinsically unstructured in their native form and are predicted to adopt an $\alpha$ helical structure upon binding to a target [16]. Their predicted molecular weight based on the size of the messages ranged from 4 to $55 \mathrm{kDa}$ in S. purpuratus [8] and from 8 to $39 \mathrm{kDa}$ in Heliocidaris erythrogramma [5], while the actual protein range as observed on western blots is between $30 \mathrm{kDa}$ to $>200 \mathrm{kDa}$ in both sea urchin species $[5,10,13]$. This difference in size is attributed to the multimerization of the Trf proteins [13]. The primary structure of the Trf proteins consists of a glycinerich region, a histidine-rich region and a C-terminal region [4]. The Gly-rich region contains the protein multimerization motif in its C-terminus, which mediates the Trf protein multimerization [14]. Recombinant peptides of the Gly-rich and His-rich Trf regions were found to have both different and overlapping antigen-binding specificities [14].

The Trf proteins were so far found to be expressed in a phagocytic subset of sea urchin coelomocytes in at least two different expression patterns: in small phagocytes, Trf proteins were localized to the cell surface and in cytoplasmatic vesicles in filopodia, whereas in large polygonal and discoidal phagocytes the proteins were localized to perinuclear vesicles $[5,13]$. On the other hand, no Trf proteins were so far localized in other cell types, including red spherule cells and the vibratile cells [21]. Immunolocalization of Trf proteins in S. purpuratus histological sections identified cellular Trf protein expression in all major sea urchin organs, including axial organ, gut, esophagus, gonad, and pharynx [11], which is attributed to infiltrated coelomocytes [21].

Based on the multiple studies in S. purpuratus, a rough model for Trf-mediated immune response was recently proposed $[15,21]$. According to the model, the Trf protein isoforms that are stored within the perinuclear vesicles of phagocytes are secreted to the coelomic fluid following the detection of bacterial challenge. The secreted Trf protein isoforms multimerize with the same/other isoforms upon binding to bacteria and may adapt an $\alpha$ helical shape. The proteins are also predicted to be involved in bacterial growth retardation [15]. It is not known how Trf-bound bacteria are recognized by the phagocytes; however, it was suggested that it may be facilitated through either an unknown receptor, multimerization with surface Trf proteins or through phosphatidic acid (PA) within the cell membranes. It was shown that Trf protein binding to PA may cause membrane deformation and therefore may participate in phagocytosis [22].

To date, the $\operatorname{Trf}$ gene/protein family was characterized in only two sea urchin species - $S$. purpuratus and H. erythrogramma. In this study, we characterize the Trf genes and proteins and the Trfmediated immune response in a third sea urchin species - the Mediterranean Sea urchin Paracentrotus lividus. P. lividus is abundant in shallow subtidal areas and in tidal pools on rocks or seagrass meadows in the Mediterranean sea and in the northeastern Atlantic ocean [23]. It is widely used in the food industry (e.g. [24]) as well as a model in scientific research, including in the fields of developmental biology (e.g. [25]) and comparative immunology (e.g. [26]). 
Our results suggest that similarly to $S$. purpuratus and H. erythrogramma, $P$. lividus utilize a diverse Trf protein repertoire as part of its immune response to pathogens. We found that the PlTrf genes and the translated protein sequences, although phylogenetically distinct from $S$. purpuratus and $H$. erythrogramma, bear the same basic structure of $S p \operatorname{Trf}$ and HeTrf genes and proteins. In this study, we used three anti-SpTrf antibodies previously raised against the peptides within the $\mathrm{N}$ terminus, the central part and the $\mathrm{C}$ terminus of the Trf proteins [13]. Using three different approaches, we first verified the cross-reactivity of these antibodies with PITrf proteins. Western blot analyses depicted high PITrf protein diversity among different $P$. lividus individuals. Furthermore, each of the sea urchins tested expressed a different Trf protein profile in the coelomocytes vs. the cell-free coelomic fluid (CF). Using FACS, we identified five different coelomocyte sub-populations with detectable membranal PITrf expression. We also showed that a challenge with heat-killed E. coli bacteria is followed by a sharp increase in the ratios of TRF-positive coelomocytes and that the E. coli-specific response is likely being mediated by a specific set of Trf proteins. Lastly, we demonstrated that $P$. lividus hemolymph plays a role in the phagocytosis of $E$. coli bacteria, a process which is inhibited by blocking membranal PITrf activity using the anti-Trf antibodies.

\section{Results}

\section{The Trf family in the Mediterenean sea urchin Paracentrotus lividus}

For the initial characterization of the Trf family in P. lividus, we obtained a total of 94 PlTrf transcript and DNA sequences from the Transcriptome Shotgun Assembly (TSA) databases HACU01, GCZS01, GEDS01 and GFRN01 (NCBI) and Octopus database (http://octopus.obsvlfr.fr/blast/oursin/blast_oursin.php), of which 70 sequences were unique after UTRs removal (Fig. S1). To understand the phylogenetic position of the PITrf sequences, twenty-five PlTrf CDS sequences of different sizes were chosen and aligned to an equal number of randomly picked full SpTrf and HeTrf CDS sequences. The alignment-based phylogenetic tree showed clustering of the Trf sequences according to species, from which they were originated with similar evolutionary distance among the clusters (Fig 1A). In contrast to the other two species, of which sequences showed homogeneous distribution, the $P$. lividus Trf cluster was sub-divided into three clades. This subdivision of PlTrf sequences may reflect segregation in function or may be caused by the limited sequence repertoire of the currently available databases from which the sequences were obtained. So far, no $\operatorname{Tr} f$ sequences have been identified in non-echinoid echinoderm classes such as sea stars (class: Asterozoa) and sea cucumbers (class: Holothuroidea) nor in basal sea urchins such as pencil sea urchin Eucidaris tribuloides (Fig. 1A), suggesting the Trf family is unique to higher sea urchins [21]. Within the echinoid lineage, in addition to Trf sequences from S. purpuratus, H. erythrogramma and P. lividus, Trf sequences were also identified in Strongylocentrotus franciscanus and Alocentrotus fragilus [27], but are not publicly available. A single $\operatorname{Trf}$-like sequence from $S$. intermedius (genebank accession: ADO13494.1) was also identified.

To characterize the $P$. lividus Trf primary protein sequences, we selected twelve full-length translated PlTrfCDS with unique sequence element content. The translated PITrf coding sequences were between 220-308 amino acids (aa) in length, which is similar to the size range of the HeTrf protein sequences (257-349 aa long) and the SpTrf sequences (276-354 aa long). All PlTrf aa sequences had the typical signatures of $\operatorname{Trf}$ sequences, including the leader sequence, glycine-rich (Gly-rich) and histidinerich (His-rich) regions and conserved sequence elements. However, the RGD motif (Arg-Gly-Asp cellbinding peptide motif), previously characterized in S. purpuratus [4], was absent from both PITrf and HeTrf protein sequences suggesting that it is not a canonical Trf motif. The translated protein sequences had a variable number of glycine residues in the Gly-rich region. The His-rich region also varied in the number of histidines, where shorter versions contained 9 histidines (element 20 in sequences PL001, PL005, PL006, PL009, PL023) and longer versions had up to 19 histidines (elements 19-20 in sequences PL10, PL011, PL015, PL018, PL021 and PL022) (Fig. 1B). An alignment of all unique translated PlTrf CDS identified 22 sequence elements determined based on the alignment gaps. The PITrf elements contained between 3 and 64 amino acids (aa). The PITrf hydrophobic leader sequences contained 21 aa, and the most common leader sequence was MELKLILIIAIVAAITISA with variations at positions 5, 7, 9 and 16 (Fig. 1B, 12 chosen sequences shown). 
A

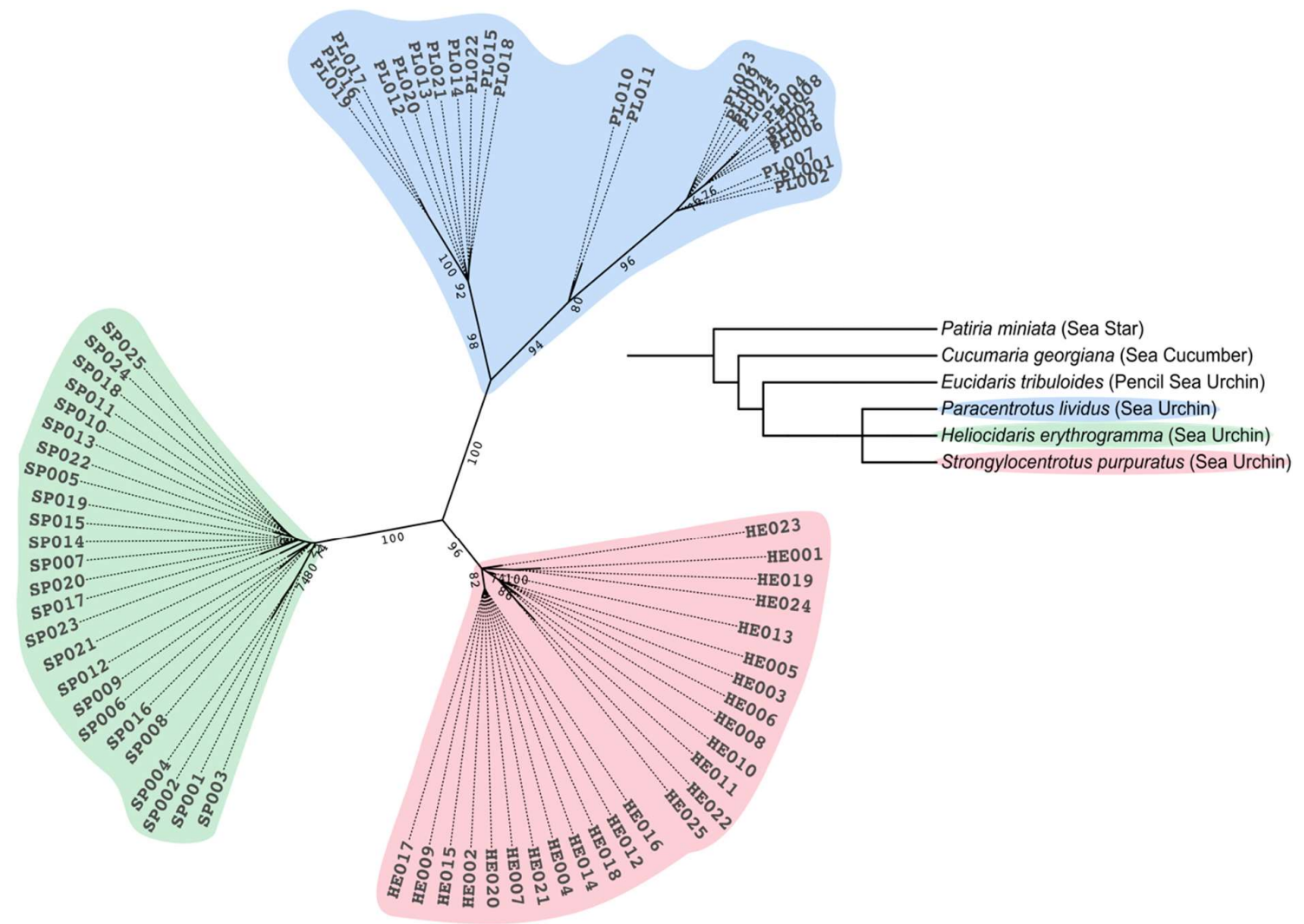

$\mathbf{B}$

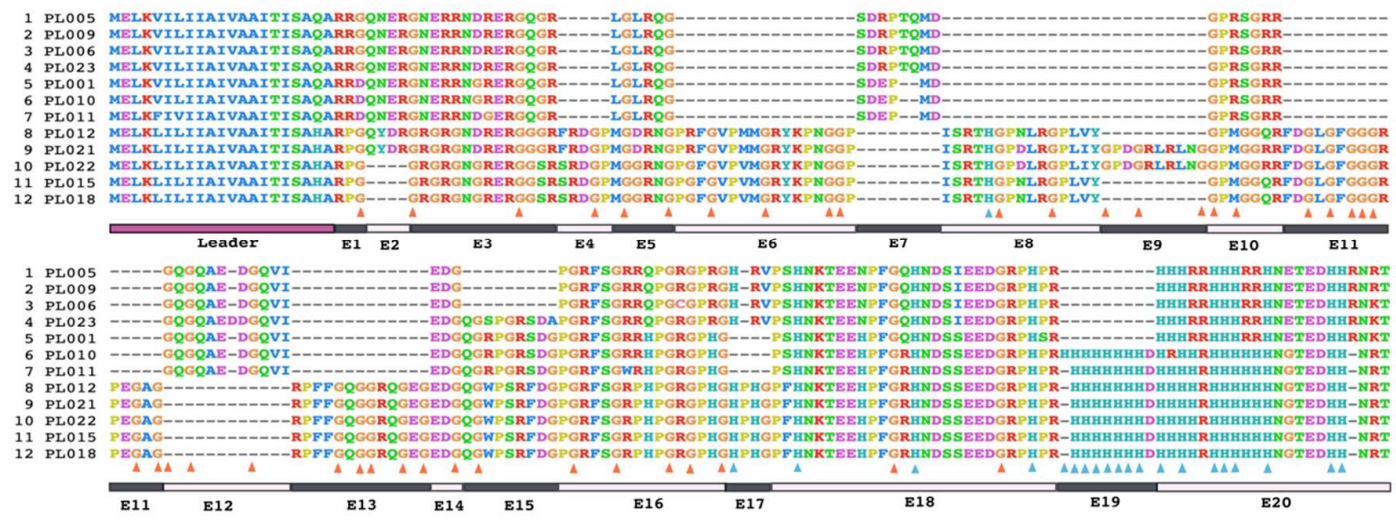

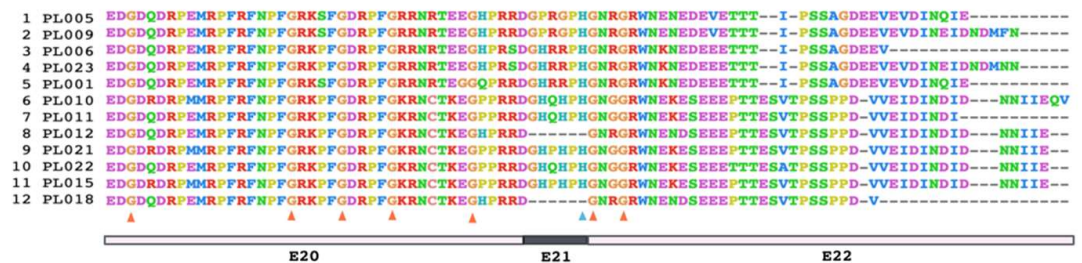

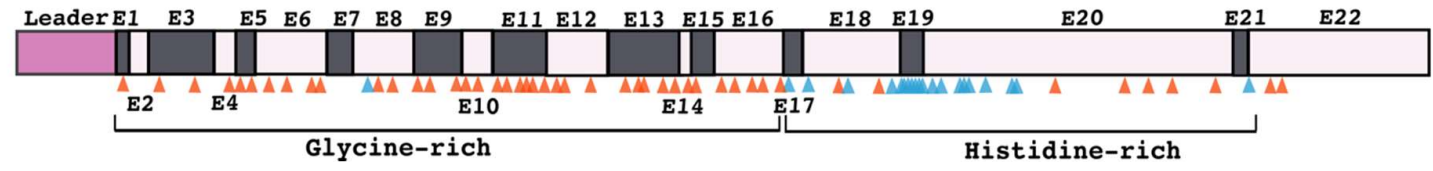

Fig. 1. The PITrf phylogeny and the structure of the translated protein sequences. A. An unrooted phylogenetic tree of unique PITrf, HeTrf and SpTrf CDS. Maximum likelihood (ML), NeighbourJoining (NJ), and Minimum Evolution (ME) analyses resulted in similar phylogenetic trees. Only the MLbased tree (Hasegawa-Kishino-Yano model [28], highest log likelihood $(-1640.98)(+G$, parameter $=$ 
2.7099)) is presented. The $\operatorname{Tr} f$ sequences were clustered according to the three sea urchin species $(P$. lividus, S. purpuratus and $H$. erythrogramma). In P. lividus, sequences were subdivided into 3 clades according to the element patterns. The whole genome-based phylogenetic tree (phyloT, NCBI taxonomy) shows evolutionary relationships among echinoderms species. The pencil sea urchin- Eucidaris tribuloides, Paritia miniata (a sea star) and Cucumaria georgiana (a sea cucumber) do not contain Trflike sequences in their genomes. B. Alignment of 12 full translated PITrf aa sequences. The sequences were aligned in MEGAX Muscle [29] with manual correction considering three or more aa surrounded by gaps as an element. The 12 sequences were chosen from all publicly available $\operatorname{PlTrf}$ sequences so they will cover all available sequence elements. Sequence alignment is shown. Bars underneath the alignment representing the leader (In purple) and the 22 sequence elements (in dark and light shades of gray and light pink). PITrf protein structure scheme summarizing the alignment is shown at the bottom. Orange triangles indicate glycine residues. Blue triangles indicate histidine residues. Sequence elements are marked as E1-E22.

\section{Anti-SpTrf polyclonal antibodies cross-bind PITrf proteins}

Polyclonal SpTrf antibodies, provided by Prof. L. Courtney Smith from the George Washington University, were originally raised against three synthetic peptides corresponding to conserved sequences of the N-terminus, central part and C-terminus of the SpTrf proteins [13]. The three peptides for which the antibodies were raised are termed "66" (AHAQRDFNERRGKENDTER), "68" (GGRRGDGEEDTDAAQQIGDGLG), and "71" (GTEEGSPRRDGQRRPYGNR) and are part of SpTrf sequence elements 1, 7 and 25 accordingly. Similar sequences (41-66\% aa identity to the 66 region, $30-40 \%$ to the 68 region and $70-88 \%$ to the 71 region) were identified in the $P$. lividus translated sequences within elements 1-3, 12-15 and 20-22 (Fig. 2A). Since their development, the SpTrf antibodies have been extensively used and became an essential tool in studying the Trf protein family. In order to use these antibodies to study the Trf family in P. lividus, as was done for H. erythrograma [5], we began with verification of its cross-reactivity with PITrf proteins by three different approaches:

We first used a Nickel (Ni)-column to enrich the PITrf proteins from whole coelomic fluid protein extract based on the binding of Ni to the multiple histidine residues of the Trf proteins. The resulting WB showed different patterns of Ni-bound vs. unbound proteins. Two major Trf protein sizes of $\sim 120$ and $\sim 50 \mathrm{kDa}$ for sea urchin \#1 vs. $\sim 250$ and $\sim 30 \mathrm{kDa}$ in sea urchin \#2 were identified in the Ni-bound fraction. In contrary, proteins mainly of $\sim 12$ and $\sim 20 \mathrm{kDa}$ in size were identified in the unbound fraction of Sea urchin \#1 and were absent from Sea urchin \#2, which had only $>250$ and several weak bands of smaller sizes in its unbound fraction (Fig. 2B). These results are similar to previous results from Nicolumn enrichment of SpTrf proteins that identified different Trf protein sizes in the Ni-bound and unbound fraction of the Ni-column assay $[6,15]$. The unbound PITrf proteins may include family members with shorter His-rich regions, such as the proteins that would be encoded based on sequences PL001, PL005, P1006, PL009 (Fig. 1B).

To test whether each of three SpTrf polyclonal antibodies $(66,68$ and 71$)$ in the mixture have a similar binding pattern, we used each of the antibodies separately in WB of coelomocytes and CF protein extract from the same individual. Except for two differences in the coelomocyte fractions of the 68 and 71 anti-SpTrf blots, the resulted WB band patterns were identical for the three antibodies, suggesting that the Trf-binding is specific. The two differences were 1) a $\sim 30 \mathrm{kDa}$ band that was present in the coelomocyte fraction of the 68 anti-SpTrf blot, which replaced the $\sim 20 \mathrm{kDa}$ band that appeared in the coelomocyte fractions identified by the two other antibodies and 2) an additional, weak $\sim 17 \mathrm{kDa}$ band that was found in the coelomocyte fraction of the 71 anti-SpTrf blot, which was not present in any of the other blots (Fig 2C). We speculated that these differences are due to the truncation and multimerization processes of specific membrane attached PITrf proteins. It was also noticed that the WB band patterns received for the three anti-SpTrf antibodies were significantly different between the coelomocytes and the CF (Fig 2C). Since this difference between the cellular and humoral Trf fractions was so far not characterized, we followed these results with further investigation using other sea urchins as described in the next section. 
A

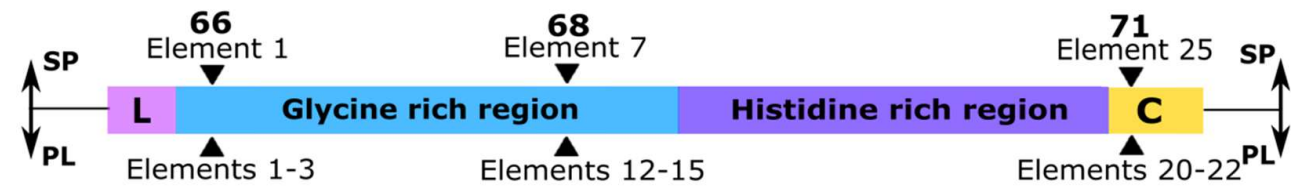

B

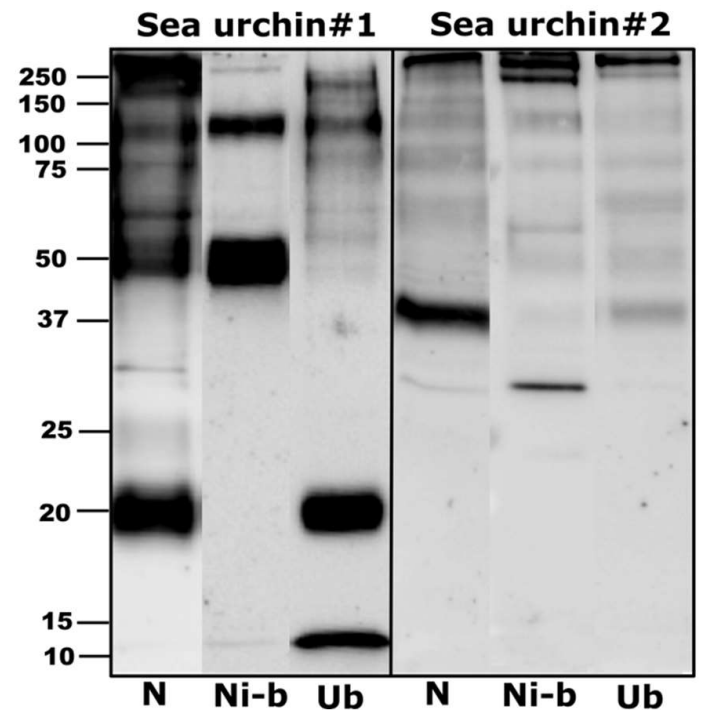

C

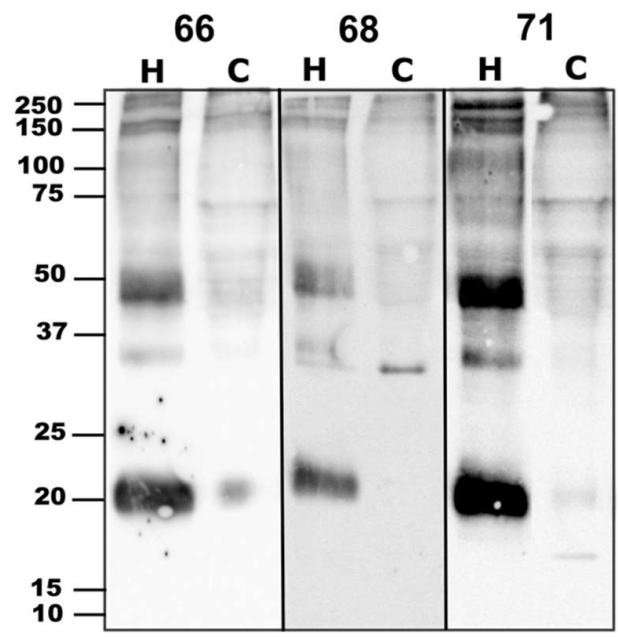

Fig. 2. Specific binding of anti-SpTrf antibodies to PITrf proteins A. Schematic structure of a Trf protein with the locations to which the three SpTrf antibodies bind. The locations for 66, 68, and 71 Trf antibodies binding are marked with black triangles for S. purpuratus (SP) and their equivalents in P. lividus (PL). B. WB of native, nickel-bound and unbound PITrf proteins with anti-SpTrf antibody mixture. Ni-Column Trf protein enrichment was performed according to [6], based on the binding of the histidine residues to the Nickel. $\mathrm{N}$ - native protein extract from the CF. Ni-b-Nickel bound proteins. Ub-Nickel column washed flow-through fraction (unbound proteins). C. WB of PITrf proteins from hemolymph and coelomocytes with each of the three antiSpTRF antibodies seperately. The western blot was made based on a whole protein extract from CF (hemolymph $(\mathrm{H})$ ) and coelomocytes $(\mathrm{C})$ of the same sea urchin. Each blot was incubated with either 66, 68 or 71 SpTrf antibodies.

In a third anti-SpTrf cross-binding verification approach, we implemented a targeted massspectrometry (MS) analysis on anti-SpTrf bound proteins from native $P$. lividus protein extracts and from the Ni-enriched protein fractions. Using the relevant WBs as a reference, SDS gel slices, an equivalent of anti-SpTrf positive high-molecular weight bands, were sent for MS. The MS analysis identified PITrf peptide sequences from both native and Ni-enriched fractions. As expected, a higher number of PITrf matches were observed in the Ni-enriched fraction compared to the whole native extract (6 vs. 4). While matches of numerous conserved proteins from different sea urchins were obtained, Trf protein matches were strictly of $P$. lividus (Table 1). The size range of the full MS-identified PITrf proteins was 239-307 aa with a predicted molecular weight of 27.2 to $34.3 \mathrm{kDa}$ and predicted uniform isoelectric points $(\mathrm{Pi})$ of $\mathrm{pH} 7.5$ to 8.8 . The presence of multiple Trf isoforms with high number of matches in the analyzed samples points with a high confidence level to the presence of the PITrf proteins in the samples from P. lividus.

Table 1. Mass spectrometry PITrf hits

\begin{tabular}{cccccccc}
\hline Accession & Name & $\begin{array}{c}\text { Coverage } \\
{[\%]}\end{array}$ & $\begin{array}{c}\text { Unique } \\
\text { Peptides** }\end{array}$ & Length aa & MW [kD] & calc. pI \\
\hline \multirow{3}{*}{ Elution* } & GEDS01012456.1 & Trf [P_lividus] & 27 & 14 & 301 & 33.7 & 7.64 \\
& GCZS01069164.1 & Trf [P_lividus] & 16 & 4 & 243 & 27.9 & 8.34 \\
& GCZS01069162.1 & Trf [P_lividus] & 4 & 2 & 241 & 27.6 & 8.46 \\
& HACU01465648.1 & Trf [P_lividus] & 6 & 2 & 244 & 27.7 & 6.87 \\
& GFRN01311978.1 & Trf [P_lividus] & 2 & 1 & 239 & 27.3 & 8.09 \\
& GEDS01012454.1 & Trf [P_lividus] & 2 & 1 & 307 & 34.3 & 8.82 \\
\hline
\end{tabular}




\begin{tabular}{llllllll}
\hline & GEDS01012454.1 & Trf [P_lividus] & 18 & 8 & 307 & 34.3 & 8.82 \\
Native* & GFRN01311979.1 & Trf [P_lividus] & 13 & 7 & 239 & 27.3 & 8.09 \\
& HACU01465651.1 & Trf [P_lividus] & 6 & 2 & 239 & 27.2 & 7.52 \\
& GCZS01069162.1 & Trf [P_lividus] & 4 & 2 & 241 & 27.6 & 8.46 \\
\hline
\end{tabular}

* Western blot bands corresponding to the gel slices used for MS analyses are presented in Fig. S1.

**Unique peptides: the number of peptides uniquely mapping to the protein.

\section{$P$. lividus coelomocytes and hemolymph have different subsets of TRF proteins}

Following our initial results showing different Trf protein profiles in coelomocytes vs. cell-free CF (hemolymph), we tested whether this pattern is consistent for other P. lividus individuals. WB with SpTrf antibody mixture resulted in different Trf profiles of the coelomocytes and hemolymph fractions of 7 sea urchins both in Trf band content and relative band intensity (given that the general protein concentrations of the coelomocyte and cell-free CF fractions samples for each animal were equalized). No consistent pattern was identified for the coelomocytes and hemolymph fractions, except for a short band of $\sim 20 \mathrm{kDa}$ that was present in the hemolymph fractions of all tested individuals but was absent or very weak in the coelomocyte samples (Fig. 3). A strong $\sim 35 \mathrm{kDa}$ band was present in five of the seven individuals in either the coelomocytes (sea urchins 3, 4, 5) or hemolymph (sea urchin 2, 7) fractions (Fig. 3). Although the PITrf proteins sampled for MS were of high molecular mass of 200-300 kDa (Fig. $\mathrm{S} 2$ ), the highest PITrf protein mass according to the MS analysis was $\sim 34 \mathrm{kDa}$ (Table 1). We, therefore, assume that all PITrf bands of larger sizes represent multimerized proteins in similar to what has been established for S. purpuratus and H. erythrograma.

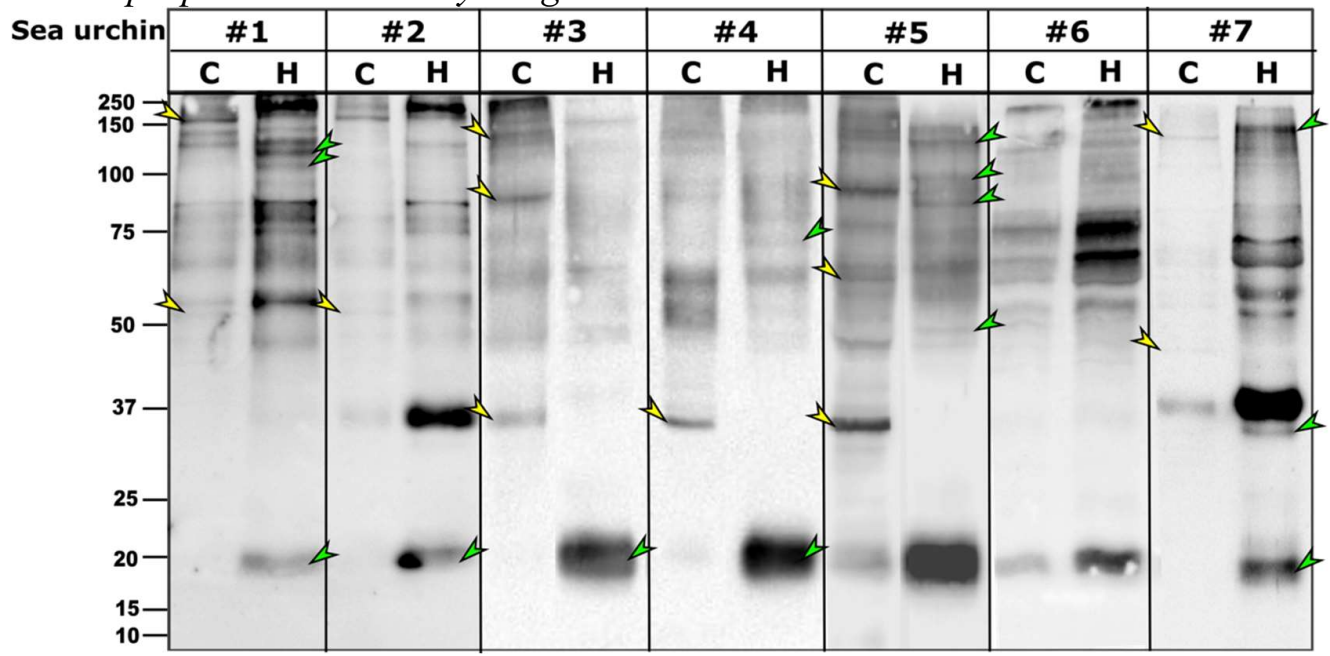

Fig. 3. Trf protein repertoires in coelomocytes and hemolymph of $7 P$. lividus individuals. $\mathrm{C}$ - coelomocytes fraction, $\mathrm{H}$ - cell-free CF (hemolymph) fraction. Multiple differences were identified between the two fractions in each of the tested sea urchins, including unique bands in the coelomocyte fractions (yellow arrowheads) and unique bands in the cell-free fractions (green arrowheads).

\section{Trf-positive cell types in $P$. lividus}

P. lividus coelomocytes are morphologically divided into phagocytes (filopodial and small), vibratile cells, red and colorless spherule cells [30], also termed amoebocytes [26]. We used FACS to sort Trf-positive coelomocytes based on their binding to the anti-SpTrf antibody mixture according to [12](Fig S4). Using fluorescent microscopy, we identified cell surface Trf proteins in five immune coelomocyte sub-populations (Fig. 4A). This is in contrary to results from S.purpurtus that detected cell surface Trf proteins only in small phagocytes, whereas red spherule cells were specifically reported to lack Trf receptors [21]. Based on their distinct autofluorescence, red cells could be separately analyzed (as performed in $[12,30,31]$ ), showing the presence of the Trf-positive red spherule cells (doublepositive for APC and FITC channels) compared to secondary antibody-only control (APC-positive only, Fig. 4B). 
A
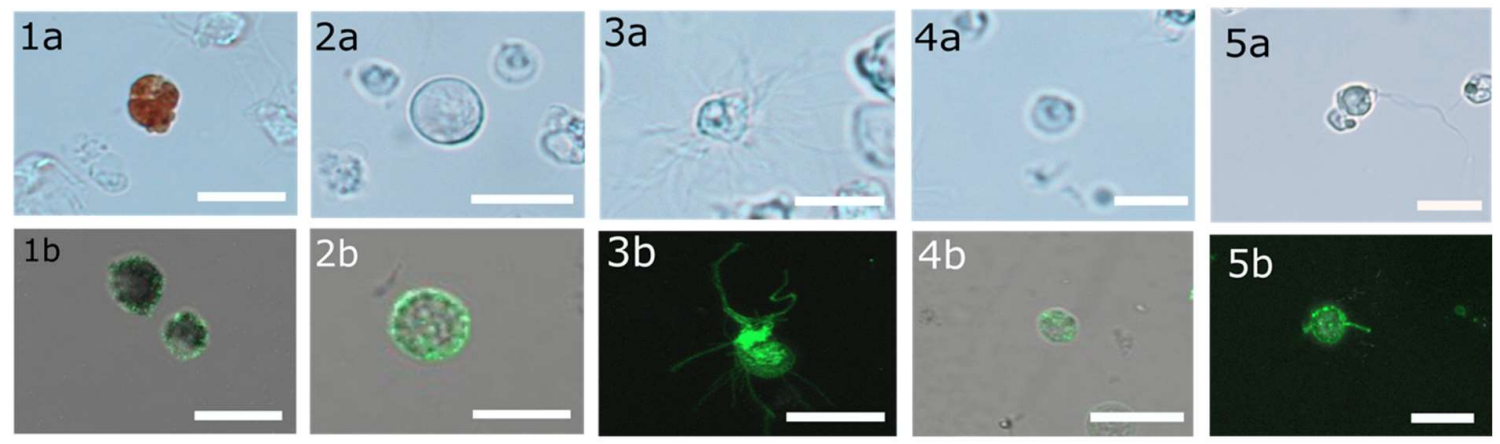

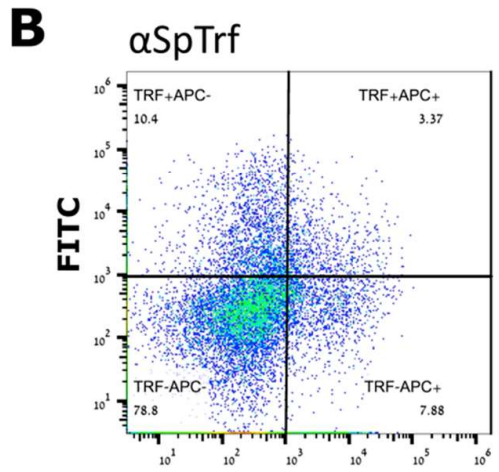

APC

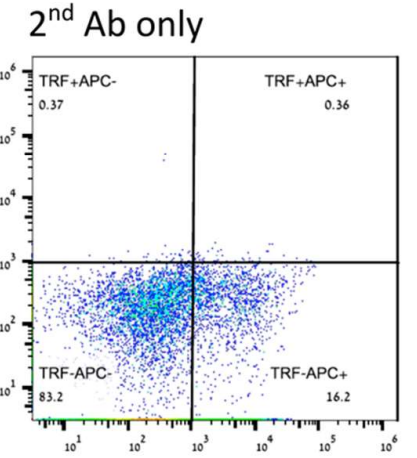

APC

Fig. 4. P.lividus Trf-positive coelomocytes. A. Live Trf-positive P. lividus cells of five coelomocyte subpopulations. a -bright field, $\mathrm{b}$ - confocal microscope imaging. Trf proteins were detected by rabbit anti-SpTrf antibodies and Alexa Fluor 488 secondary anti-rabbit antibody. Trf-positive coelomocytes include: 1. red spherule cells, 2. petaloid or small phagocytes, 3 . filopodial phagocytes, 4 . white spherule cells and 5. vibratile cells. Scale bars $=10 \mu \mathrm{m} \mathrm{B}$. Trf-positive red spherule cells sorting. The APC-positive cells are the Red spherule cells. In the $\alpha$ SpTrf treated sample (left) - The APC/FITC double-positive cells (upper right corner) are the Trf-expressing Red spherule cells. The FITC-positive/APC-negative cells (Upper left corner) represent all other SpTrf-positive cells. In the $2^{\text {nd }} \mathrm{Ab}$ only control (right) coelomocytes were incubated with the Alexa Fluor 488 secondary antirabbit antibody only. Almost no FITC-positive cells were observed.

\section{$P$. lividus Trf-expressing coelomocytes ratios increase in response to $E$.coli but not to LPS or sea urchin pathogen $V$. penaeicida challenge}

We previously showed that the $S$. purpuratus Trf-positive coelomocytes ratios increase following the challenge with heat-killed $V$. diazitrophicus bacteria [12]. In this study, we characterised the changes in the $P$. lividus Trf-positive coelomocyte ratios following challenge with heat-killed $E$. coli bacteria, LPS, and heat-killed $P$. lividus pathogenic bacteria $V$. penaeicida in comparison to sterile artificial $\mathrm{CF}$ $(\mathrm{aCF})$ injections. Although sea urchins were kept in isolated aquaria for more than 6 months before the experiment, their basal levels of Trf-positive coelomocyte ratios were highly variable (13-65\% of the total coelomocyte counts). In the first experiment, $10^{6}$ heat-killed $E$. coli bacteria or $1 \mu \mathrm{g}$ of LPS per $\mathrm{ml}$ of $\mathrm{CF}$, diluted in $100 \mu \mathrm{l}$ of sterile aCF or equivalent volumes of aCF as control, were injected to corresponding sea urchin groups of three individuals each. Coelomocytes were collected for FACS analysis at day 0 , just before the injection, and at days 1,2, 13 and 43 post-injection. A significant (p value $<0.05)$ sharp increase in the Trf-positive coelomocyte ratios was observed in response to the $E$. coli challenge after the first day, which lasted up to day 43 post-challenge. On the contrary, the aCFinjected sea urchin controls and LPS-injected sea urchins showed a decrease in the Trf-positive coelomocyte ratios one and two days post-challenge followed by an increase in ratios toward the basal levels in day 13 and 43 post-injection (Fig. 5A). We assumed that the decrease in the Trf-positive coelomocyte ratios compared to the basal levels was possibly due to the insufficient time for the coelomocyte to differentiate into Trf-expressing cells after removal of substantial $0.2-0.4 \mathrm{ml}$ of sea urchin CF from their coelomic cavity. We therefore adopted a different experimental design, which 
included CF sampling starting from day 2 to allow enough time for Trf-expressing coelomocytes to accumulate. Indeed, when we followed this design, we did not observe a decrease in the Trf-expressing coelomocytes population in the unchallenged sea urchin controls. On the contrary, the injection of aCF in the control group resulted in a mild increase the Trf-expressing coelomocytes ratios (Fig. 5B). This suggests that the decrease in Trf-positive cells in the first experiment was not related to the injections but rather to the timing of the sampling. We further assume that the time needed for Trf-expressing cell progenitors to differentiate after depletion due to sampling was between one and two days. In the second experiment, we tested the Trf-mediated cellular response to $V$. penaeicida, which is considered a possible $P$. lividus pathogen [32]. $10^{6}$ heat-killed $V$. penaeicida cells for $1 \mathrm{ml}$ of sea urchin coelomic fluid and equivalent volumes of aCF as a control were injected in the two corresponding groups of five sea urchins each. No significant difference was observed between the challenged sea urchins and the control group over the time course of the experiment (Fig. 5B). In both groups, a moderate increase in Trf-expressing coelomocytes ratios was observed on day 2 followed by a significant ( $p$ value $<0.05$ ) decrease to the basal levels in day 14 and another unexplained increase on day 30. Additionally, a distinct, highly Trfpositive cell population appeared in the flow cytometry analysis of day 2 in most of the experiment and control samples (Fig. S3), which was not present in days 14 and 30, suggesting Trf-based short-time response to both bacteria and $\mathrm{aCF}$ injections.

A

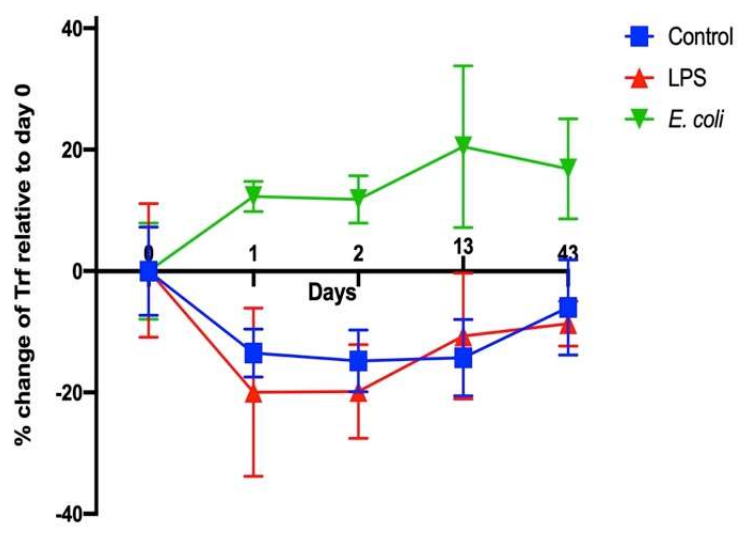

B

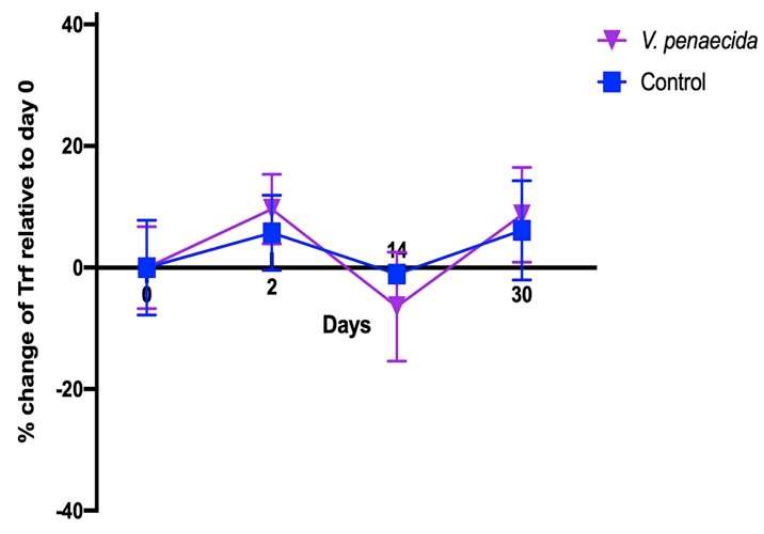

Fig. 5. Percent change of Trf-positive cells in response to a challenge with different immune elicitors. Due to the high variability in the basal levels of the Trf-positive coelomocytes within the sea urchin population, only the changes in the ratios relative to day 0 are presented. A. Trf-positive coelomocytes percent change in response to injections of heat-killed $E$. coli, LPS and aCF (control). Sampling and measurements were subsequently done on days 1,2, 13 and 43 post-injection. A significant increase in the percentage of Trf-positive coelomocytes was observed following the challenge with heat-killed E. coli over the course of the experiment. B. Trf-positive coelomocytes percent change in response to injections of heat-killed $\mathrm{V}$. penaeicida and aCF (control). Experiment and control groups did not show significant differences.

\section{Specific subset of Trf proteins bind live $E$. coli bacteria}

Quite a few $S$. purpuratus studies demonstrated specific Trf-mediated responses to different types of immune elicitors, including the expression and binding of specific subsets of Trf proteins $[4,7,8,15$, 33]. Following the strong PITrf cellular effector arm reaction to the E. coli challenge, we tested the profiles of the Trf proteins that specifically bind to live E. coli. We used the cellular and cell-free CF (hemolymph) fractions of coelomic fluid extracted from three sea urchins. WB analysis of the E. colibound proteins showed a conserved band pattern in both coelomocytes and hemolymph fractions of the tested sea urchins after 30 minutes incubation with live E. coli. Most of the E. coli-bound Trf proteins were confined within $5-9$ bands of $\sim 30$ to $\sim 60 \mathrm{kDa}$ (Fig.6). The E. coli-bound protein band sizes were absent from the untreated protein extract taken from the same three sea urchin individuals, suggesting they represent diverse Trf monomers or dimers that may be bound to E.coli molecules. 


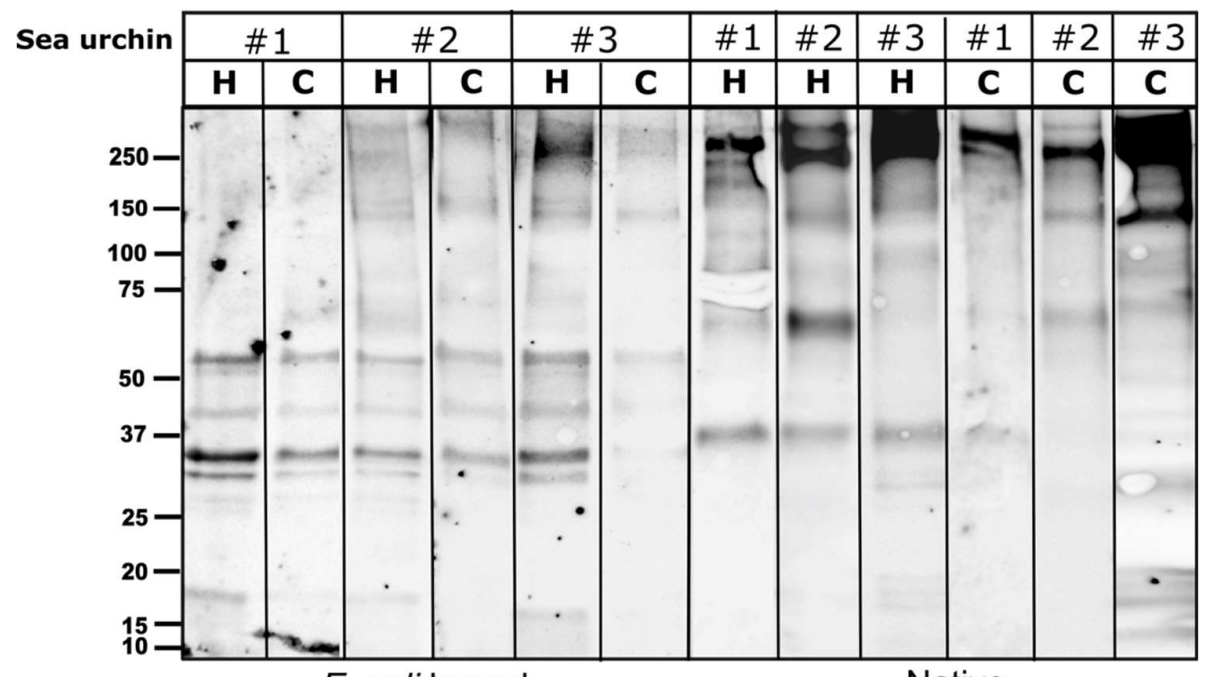

Fig. 6. Specific WB band pattern of $\boldsymbol{E}$. coli bound PITrf proteins. A similar Trf protein band pattern was observed in both coelomocytes $(\mathrm{C})$ and hemolymph $(\mathrm{H})$ from three sea urchins that were incubated with live $E$. coli bacteria. The band pattern of the untreated coelomocytes and hemolymph protein extract counterparts are shown on the right side of the figure.

\section{Phagocytosis in $P$. lividus is mediated through the hemolymph and is partially inhibited by blocking surface PITrf protein activity}

Two morphological types of phagocytes were described in P. lividus -filopodial and petaloid phagocytes $[26,34]$. The two phagocyte types are similar in size and contain long microfilamental cytoplasmatic projections of different morphology. We performed phagocytosis assays using E.coli in similar to [15]. Fluorescent bacteria were identified inside both P. lividus phagocyte types, the petaloid phagocytes were observed mostly with a single internalized bacterial cell, whereas filopodial cells mostly included multiple (up to 15) bacteria (Fig. 7A). The ratios of the phagocytes with internalized $E$. coli within the general coelomocyte population were between 1 and $3 \%$. The hemolymph in many invertebrates plays a major role in augmenting phagocytosis. Accordingly, our results show almost twofold higher phagocytosis rates by $P$. lividus phagocytes of bacteria that were pre-incubated with hemolymph compared to non-incubated ones (Fig. 7B, treatment A vs. B). It was demonstrated that the Trf proteins augment the phagocytosis of bacteria in S. purpuratus [15]. To test whether the membranebound Trf proteins participate in mediating phagocytosis in P. lividus, we used the anti-SpTrf antibody mixture to block the cell surface Trf activity. Indeed, phagocytosed bacteria were found in significantly $(\mathrm{p}<0.05)$ lower ratios in the anti-Trf pre-treated coelomocytes (Fig. 7B, treatment C) compared with the equivalent untreated coelomocytes of the same sea urchins $(n=3)$ (Fig. 7B, treatment $\mathrm{A})$. This is in spite of the pre-incubation of the bacteria with the hemolymph in both treatments.

A
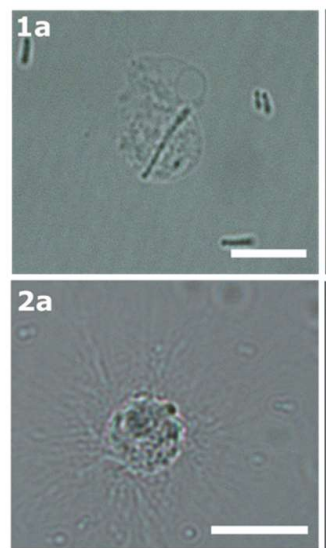

B

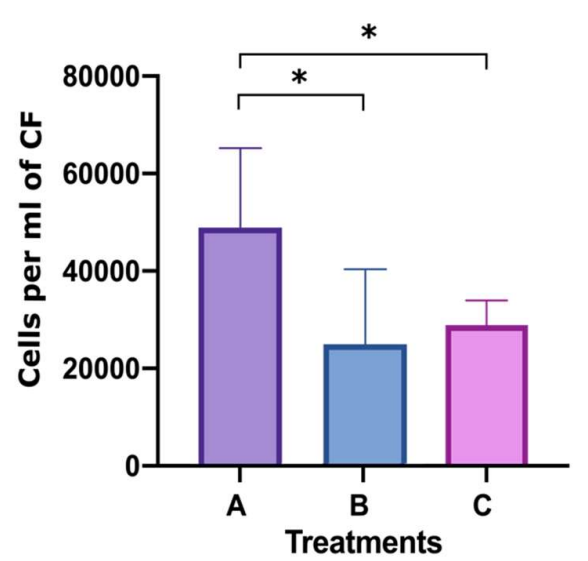

Fig. 7. Phagocytosis of $E$. coli bacteria by $P$. lividus phagocytes and its inhibition with anti-SpTrf antibody. A. P. lividus sea urchin phagocytes with internalized $E$. coli bacteria. 1a-b. petaloid phagocyte in the process 
with internalized FITC-stained $E$. coli bacteria. 2a-b. filopodial phagocyte with multiple mCherry-expressing $E$. coli bacteria in its cytoplasm. Scale bars $=10 \mu \mathrm{m}$. B. Concentrations of $\boldsymbol{P}$. lividus coelomocytes containing $\boldsymbol{E}$. coli bacteria under different treatments. A. phagocytosis ratioes in coelomocytes that were incubated with bacteria that were pre-incubated with hemolymph, B. phagocytosis ratios in coelomocytes that were incubated with untreated bacteria C. phagocytosis ratios in coelomocytes that were pre-incubated with anti-SpTrf antibodies and incubated with bacteria that were pre-incubated with hemolymph. Vertical bars indicate standard errors. Differences in phagocytosis ratios in different treatments were significant by Anova two-factor analysis. Anova single factor analysis indicated that treatment A resulted in significantly higher phagocytosis ratios compared with treatments B and $\mathrm{C} .{ }^{*}$ indicates a significant difference $(\mathrm{p}<0.05)$.

\section{Discussion}

Immune systems are probably the most diverse systems in multicellular organisms, both among species as well as within a specific population, and even among different cells of the same individual. This is especially true for invertebrates that use many different ways to achieve protection against a great variety of pathogens in their surrounding (e.g. [35]). The Trfs are an example of unique proteins that participate in a specialized immune response in sea urchins. To date, this family was studied in $S$. purpuratus as well as in H. erythrogramma. In this study, we characterize, for the first time, a family of Trf genes and proteins in the Mediterranean Sea urchin P. lividus, a known echinoid model organism. Our results suggest that despite the differences among PITrf, SpTrf and HeTrf gene and protein sequences, the basic features and the mechanism of action seem to be conserved. The PlTrf protein sequences included specific features like size, typical regions including the characteristic hydrophobic leader, glycine-rich and histidine-rich regions, distinguished element patterns, and repeats that were characterized for the two other species. On the other hand, the RGD cell-binding motif seems to be absent from both $P$. lividus and H. erythrogramma and therefore may have a specific role only in $S$. purpuratus. To be able to study the Trf-mediated response in P. lividus, we had to establish a strong molecular tool, which is sometimes hard to find for such a non-classical invertebrate immunity model. We, therefore, invested resources to validate the cross-reactivity of a potent anti-Trf antibodies mix that was developed by the Smith group against conserved areas of the SpTrf proteins [13]. We used the validated antibodies mix to characterize the PITrf protein repertoire in cells and hemolymph of different individuals, for detection of PITrf-expressing coelomocytes, FACS assays, and for the blocking of the cell surface PITrf activity in functional phagocytosis assays.

As in other sea urchin species, the PITrf proteins vary significantly among individuals, although they often share subsets of proteins of the same molecular weight. It was previously established that some SpTrf proteins multimerize through the glycine-rich Trf protein region in order to function [14]. The PITrf protein multimers vary greatly in size, indicating several levels of multimerization. The Trf protein multimerization is irreversible and stable in high temperature, 2-Mercaptoethanol, and SDS treatments and that is why multimers are observed in WB. We identified two size categories of PITrf protein monomers of approximately 20 and $35 \mathrm{kDa}$ that are similar to the sizes of the SpTrf and HeTrf protein monomers. While the $\sim 34 \mathrm{kDa}$ monomer size was predicted based on the CDS sequences, the $\sim 20 \mathrm{kDa}$ monomer could not be predicted by the available full primary sequences and may represent truncated proteins. In S. purpuratus, truncated Trf sequences that were lacking the histidine-rich region, were referred to as potential surveillance proteins [6]. We think that this is the case with the PITrf as it is also supported by the Ni-column isolation, in which the $\sim 20 \mathrm{kDa}$ proteins were found in the unbound fraction of one of the tested sea urchins (Fig. 2A).

Recent works about the function of the SpTrf proteins suggested that they work through both cellular and humoral effector arms in augmenting phagocytosis [15, 22]. In this study, we identified significant differences between the PITrf protein repertoires of the coelomocytes (cellular) and the cellfree CF or hemolymph (humoral) fractions of the coelomic fluid in each of the tested sea urchins. The differences were observed in the monomers and the multimers content of the two fractions. The smaller PITrf monomers of $\sim 20 \mathrm{kDa}$, that were consistently present in the hemolymph, were either fully or almost absent from coelomocytes. On the other hand, the $\sim 35 \mathrm{kDa}$ monomers were present in either coelomocytes, hemolymph or in both, depending on the genotype. Significant variability was observed in the PITrf multimers content in all tested sea urchins. To better understand the functionality of each of these Trf-mediated effector routes and the possible interplay between them, we studied each of the two 
fractions separately and tested how the absence or inhibition of each of the fractions is effecting the phagocytosis process.

In contrary to the cell surface localization of Trf proteins which was restricted to small phagocytes (petaloid coelomocytes) in S. purpuratus [21], we identified at least five morphological subpopulations of coelomocyte with cell surface-bound PITrf proteins. These differences between $S$. purpuratus and P. lividus may be due to differences in Trf activity between the species. However, they may also be due to the use of FACS to sort live coelomocytes, which enabled sensitive detection prior to imaging. An increase in membranal Trf-expressing coelomocyte ratios was recorded in S. purpuratus in response to LPS [13] and $V$. diazotrophicus $[7,12]$. We followed a similar experimental design to first test the effect of LPS and heat-killed E. coli bacteria. We were surprised to see that differently than the results obtained from $S$. purpurtus, LPS did not influence the ratio of PITrf-expressing coelomocytes. On the other hand, a sharp increase was recorded from day 1 and up to day 13 E. coli post-challenge, suggesting that LPS might not be the main targets of the PITrf proteins but rather different molecules on $E$. coli surface. We also used heat-killed $V$. penaeicida, a $P$. lividus pathogen, in similar to $V$. diazotrophicus, which was used for the $S$. purpurtus immune challenge. Unexpectedly, $V$. penaeicida did not have an effect on the PITrf expressing coelomocytes, suggesting it may escape the surveillance of the PITrf proteins.

Specificity is a key feature in immune systems since it enables optimal recognition of pathogens within a variable environment and prevents self-distraction and autoimmunity [36]. Although this attribute is classically linked with adaptive immunity, a high degree of specificity may characterize certain types of innate immunity and innate immune receptors. The great protein diversity of the Trf family within the sea urchin population [10] and the unique single-gene expression in individual coelomocytes [7] allow a high degree of specificity. Indeed, SpTrf specificity was demonstrated in the specific gene-expression response to different elicitors $[6-8,15]$, the specific binding of a recombinant SpTrf protein [14] and the selective PITrf-mediated cellular response to different types of immune challenges, as is shown here. By incubating live E. coli with coelomocytes or hemolymph, we profiled the specific E. coli bound PITrf proteins. The E. coli bound PlTrf protein profiles included the same specific sets of PITrf proteins, regardless of the genotype or the coelomic fluid fraction they were identified in.

In the last experiment, we studied the role of the Trf-mediated cellular and humoral fractions in the phagocytosis of E. coli. Although it was demonstrated that SpTrf proteins augment phagocytosis [15], it was still not clear what is the function of the membranal Trf proteins in this process. We found that although the coelomocytes alone (without hemolymph) are sufficient for phagocytosis, the absence of hemolymph (humoral fraction) reduces phagocytosis efficiency. We also used the anti-Trf antibody mix to block the cell surface PITrf proteins and test whether this will affect phagocytosis efficiency. Our results demonstrate that the inhibition of membranal PITrf activity results in significant inhibition of phagocytosis.

Taken together, this study characterizes for the first time, a diverse Trf gene/protein family in the Mediterranean Sea urchin P. lividus. It demonstrates the collaboration of distinct cellular and humoral Trf-mediated effector arms in mounting specific P. lividus immune responses, opsonization of bacteria and phagocytosis.

\section{Materials and methods}

\section{Alignment and phylogenetic analyses of Trf sequences}

The search for PlTrf sequences was performed in 4 TSA databases: HACU01, GCZS01, GEDS01, GFRN01 and in the Octopus database (http://octopus.obs-vlfr.fr/blast/oursin/blast_oursin.php). $S$. purpuratus and $H$. erythrogramma Trf sequences were compared to the databases using local BLAST algorithm. For the initial characterization of the Trf family in P. lividus, we obtained a total of 94 PlTrf transcript and DNA sequences, of which 70 were sequences with unique full open reading frames after the removal of UTR sequence edges. 25 sequences from each of the three species were aligned using ClustalW multiple alignments with default parameters and further manual refinement using MEGAX [29]. Phylogenetic analyses of PITrf sequences were performed using Maximum Parsimony, Neighborjoining and Maximum Likelihood analyses with bootstrapping of 100 repetitions. The best algorithm 
combination was inferred by MEGAX as Maximum Likelihood with Hasegawa-Kishino-Yano model [28]. Initial trees for the heuristic search were obtained by applying the Neighbor-Joining method to a matrix of pairwise distances estimated using the Maximum Composite Likelihood (MCL) approach. A discrete Gamma distribution was used to model evolutionary rate differences among sites (3 categories $(+G$, parameter $=2.7099))$. The tree was visualized as unrooted with bootstrap value displayed in ITOL program [37]. Twelve translated PlTrf sequences were chosen based on their unique aa sequence to generate alignments and to represent all available element patterns.

\section{Sea urchins}

Sea urchins were obtained from the Israeli National Center for Mariculture in Eilat. Urchins were kept in artificial seawater (Red Sea Fish Pharm Ltd.) in a 165-liter aquarium in the Molecular Ecology Laboratory at the Ariel University, of which $1 / 10$ was replaced every week. The water salinity was kept around $40 \mathrm{ppt}$ and the temperature between 20 and $22^{\circ} \mathrm{C}$. The animals were fed once a week with either fresh or frozen Ulva algae. Unhealthy-looking or dying sea urchins are isolated to a different aquarium to avoid contamination of healthy individuals. Experiment animals were isolated in individual floating plastic cages in a separate compartment in the aquarium.

\section{Bacteria culturing}

Escherichia coli (25922 strain) and mCherry-expressing E. coli were kindly provided by prof. Shiri Navon-Venezia and Ms. Helena Tuchinsky, Vibrio penaeicida (51842 strain) were obtained from ATCC bioresource center (USA), Commercial lipopolysaccharide (LPS) from E. coli was purchased from Sigma-Aldrich (Israel). E. coli bacteria were cultured in liquid Luria Bertani (LB) medium overnight at $37^{\circ} \mathrm{C}$. $V$. penaeicida bacteria were cultured in Marine Broth media (MB) from SigmaAldrich (Israel) overnight at $26^{\circ} \mathrm{C}$. The concentration of bacteria was either calculated based on OD values measured in a Nanodrop NP80 spectrophotometer (Implen) or manually counted with a hemocytometer. All bacteria were heat-killed for $5 \mathrm{~min}$ at $95^{\circ} \mathrm{C}$ and then washed and resuspended in aCF (10 mM CaCl2, 14 mM KCl, 50 mM MgCl2, 398 mM NaCl, 1.7 mM Na2HCO3, 25 mM Na2SO4) and used shortly after in the different experiments.

\section{Preparation of coelomocytes and cell free CF samples}

To study the $P$. lividus Trf-mediated immune response, $\sim 300 \mu \mathrm{l}$ of coelomic fluid was collected from the coelomic cavity of the sea urchin with a $23 \mathrm{G}$ syringe filled with the same volume of cold calcium-magnesium free seawater CMFSW-EH (460 mM NaCl, $10.73 \mathrm{mM} \mathrm{KCl,} 7.06 \mathrm{mM} \mathrm{Na} 2 \mathrm{SO}$, $2.38 \mathrm{mM}$ NaHCO3, $70 \mathrm{mM}$ EDTA, $20 \mathrm{mM}$ HEPES; $\mathrm{pH}$ 7.4) and kept on ice until processing. The CF was then centrifuged at $500 \mathrm{~g}$ at $4{ }^{\circ} \mathrm{C}$ for $5 \mathrm{~min}$. The supernatant was carefully collected without disturbing the cell pellet and transferred to a separate tube. The supernatant was centrifuged again at the higher speed of $2000 \mathrm{~g}$ at $4{ }^{\circ} \mathrm{C}$ for $5 \mathrm{~min}$, to obtain the cell-free CF. The cells (the pellet of the first centrifugation) were carefully resuspended and washed in CMFSW-EH. The cell-free CF and the hemolymph-free coelomocytes were separately used for WB and phagocytosis assays.

\section{Protein extraction}

The whole coelomic fluid, the cell-free CF and the coelomocytes were separately lysed using RIPA buffer (Thermo Fisher Scientific, USA). The lysate was incubated for $30 \mathrm{~min}$ at room temperature with protease inhibitor cocktail III (Calbiochem) with constant vortexing. Alternatively, cells were sonicated for $5 \mathrm{~min}$. Cell debris was pelleted by centrifugation for $10 \mathrm{~min}$ at $12,000 \mathrm{rpm}$ at $4{ }^{\circ} \mathrm{C}$. The supernatant was stored as sample aliquots at $-80^{\circ} \mathrm{C}$ until use. To equalize the protein quantities before loading on the WB gels, protein concentrations were measured using Bradford assay and with an NP80 spectrophotometer (Implen).

\section{His60 Ni-column binding assay}

The His60 Ni-column binding assay was performed according to [6]. The whole coelomic fluid was mixed with $2 \mathrm{~mL}$ of lysis buffer (50 mM NaH2PO4, $300 \mathrm{mM} \mathrm{NaCl}, 10 \mathrm{mM}$ Imidazole, adjusted pH to 8.0 with $\mathrm{NaOH}$, supplemented with $1 \mathrm{mg} / \mathrm{ml}$ lysozyme). The mixture was sonicated for 5 minutes and incubated at room temperature for $10 \mathrm{~min}$. The lysate was centrifuged for $30 \mathrm{~min}$ at $10,000 \mathrm{~g}$ at $4{ }^{\circ} \mathrm{C}$, and the supernatant was collected. $2 \mathrm{~mL}$ of His60 Ni Superflow Resin (Takara Bio, Japan) was added to a gravity column, followed by $10 \mathrm{~min}$ incubation with $4 \mathrm{~mL}$ equilibration buffer $(50 \mathrm{mM} \mathrm{NaH} 2 \mathrm{PO} 4,300$ $\mathrm{mM} \mathrm{NaCl}, 10 \mathrm{mM}$ Imidazole in water, adjusted $\mathrm{pH}$ to 8.0 with $\mathrm{NaOH}$ ). The lysate supernatant was added to the column and incubated with resin for $1 \mathrm{~h}$ at $4^{\circ} \mathrm{C}$ with gentle shaking after which it was drained and 
discarded. Unbound proteins were collected with 4 volumes of wash buffer (50 mM NaH2PO4, $300 \mathrm{mM}$ $\mathrm{NaCl}, 30 \mathrm{mM}$ Imidazole in water, Adjusted $\mathrm{pH}$ to 8.0 with $\mathrm{NaOH}$ ) that ran through the column. Nibound proteins were then eluted with 5 volumes of elution buffer $(50 \mathrm{mM} \mathrm{NaH} 2 \mathrm{PO} 4,300 \mathrm{mM} \mathrm{NaCl}$, $500 \mathrm{mM}$ Imidazole, water, Adjusted $\mathrm{pH}$ to 8.0 with $\mathrm{NaOH}$ ). The washed (unbound) and eluted proteins were further used for WB and MS.

\section{Western blots}

Samples with equal quantities of whole protein extracts were mixed with X4 Laemmli sample buffer (Bio-Rad). The samples were then incubated at $90^{\circ} \mathrm{C}$ for $5 \mathrm{~min}$, and 10-30 $\mu \mathrm{g}$ of protein was loaded on $10 \%$ or $12 \%$ SDS-PAGE gels. Gel electrophoresis was performed in a fresh running buffer (10 x $25 \mathrm{mM}$ Tris, $192 \mathrm{mM}$ glycine, 0.1\% SDS, pH 8.3). Gels were subjected to semi-dry transfer with Trans-Blot Turbo ${ }^{\mathrm{TM}}$ System (Bio-Rad) onto commercial PVDF membranes (Bio-Rad) with the preprogrammed Bio-Rad $1.5 \mathrm{~mm}$ gel transfer program. The membranes were blocked in BSA-TBST blocking solution (5\% BSA in $20 \mathrm{mM}$ Tris, $150 \mathrm{mM} \mathrm{NaCl}, 0.1 \%$ Tween 20 ) for $1 \mathrm{~h}$. Blocking solution was then replaced with $2.5 \%$ BSA-TBST solution containing 1:10000 of primary Rabbit anti-SpTrf antibodies and kept overnight at $4{ }^{\circ} \mathrm{C}$. The membranes were washed 5 times for 5 min with TBST and then incubated in TBST containing 1:20000 HRP Goat anti-Rabbit antibody (Abcam) for 1h. Pictures were obtained by Quantity One or with ChemiDoc imaging systems (Bio-Rad) and processed with ImageJ (FIJ) or the Image Lab (Bio-Rad) softwares.

\section{Mass spectrometry}

The nickel-bound (eluted) and washed protein fractions from the Nickel column were run in two identical batches on an SDS-PAGE gel. The gel was cut according to the batches. The first gel half was used for the identification of the band sizes by WB. The second gel half was used to cut gel slices that corresponded to high molecular Trf-positive bands that were identified in the WB. An empty gel slice from the gel corner was used as a background control. Gel slices were further processed for MS as follow: The bands were subjected to in-gel tryptic digestion, followed by a desalting step. The resulting peptides were analyzed using Waters HSS-T3 column on nanoflow liquid chromatography (nanoAcquity) coupled to high resolution, high mass accuracy mass spectrometry (Q Exactive Plus). The samples were analyzed on the instrument in a discovery mode. The data was processed using Proteome Discoverer version 2.4.1.15, searched against custom databases, to which a list of common lab contaminants was added. The search was done with the Byonic search algorithms using the following modifications: fixed modification of cysteine carbamidomethylation, and variable modification of methionine oxidation, asparagine- or glutamine-deamidation, and protein N-terminal acetylation. One combined database was generated from the custom tailored subjected databases: Euechinoidea_proteins_collective (53221 protein entries of all identified to the date proteins for Euechinoidea subclass uploaded from NCBI), P_lividus_translated_EMBOSS (raw translated by all 6 frames peptides untreated while removing all ' $X$ ' and '*' signs of the stop codons) (468 entries), Trf_P_lividus_cleaned (53 entries of cleaned manually confirmed sequences).

\section{Bacterial binding essay}

For characterizing the cell-free $\mathrm{CF}$ and coelomocyte $\operatorname{Trf}$ protein repertoire that binds live $E$. coli bacteria we performed a binding assay. $10^{9}$ live E. coli was incubated with either live P. lividus coelomocytes or cell-free $\mathrm{CF}$ for $30 \mathrm{~min}$ at room temp with gentle shaking. After the incubation, coelomocytes were washed 3 times for 5 min with CMFSW-EH. Bacteria that were incubated with cellfree $\mathrm{CF}$ were pelleted at $10000 \mathrm{~g}$ for $5 \mathrm{~min}$ and washed 2 times for $5 \mathrm{~min}$. Both coelomocytes and bacteria were lysed and used for Trf protein profiling by Western blot as described above.

\section{Immune challenge}

For the immune challenge, we injected $100 \mu \mathrm{laCF}$ containing $10^{6}$ heat-killed $\left(95^{\circ} \mathrm{C}\right.$ for $\left.5 \mathrm{~min}\right) \mathrm{E}$. coli or $V$. penaeicida bacteria or $1 \mu \mathrm{g}$ of LPS per $\mathrm{ml}$ of sea urchin CF. The CF volume was calculated according to (weight of animal $(\mathrm{g}) \times 0.22=\mathrm{ml}$ whole coelomic fluid $(\mathrm{wCF})$ ) [38]. Equivalent volumes of aCF were injected in the control animals. Before the injection untreated animals CF was taken for measuring the base level of Trf expression.

\section{FCM and FACS}

For flow cytometry (FCM) analysis, we used challenged and unchallenged animals at day 0,1 , 2, 14, 30, 43 post-challenge. The coelomic fluid was collected as described above. All steps were 
performed on ice. Cells were counted and adjusted to $10^{6}$ cells $/ \mathrm{ml}$. Coelomocytes were washed as described above and pelleted by centrifugation at $500 \mathrm{~g}$ for $5 \mathrm{~min}$ at $4^{\circ} \mathrm{C}$. The pellet was gently resuspended in $100 \mu \mathrm{l}$ of a staining medium (3.3X PBS with $20 \mathrm{mM}$ HEPES, $2 \%$ fetal calf serum and $0.09 \%$ Sodium Azid-NaN3, pH 7.4) containing 1:100 concentration of three polyclonal SpTrf antibodies mixture provided by prof. L. Courtney Smith (1:300 of each polyclonal antibody). Control samples were resuspended in a staining medium without the antibody. Samples were incubated for $30 \mathrm{~min}$ on ice in the darkness to allow antibody binding. Then cells were pelleted again, resuspended, and washed in $500 \mu \mathrm{l}$ staining medium. The washed cells were resuspended in $30 \mu \mathrm{l}$ of staining medium with the 1:250 goat anti-rabbit secondary antibody conjugated to Alexa Fluor 488 (Thermo Fisher Pierce) and incubated on ice for $30 \mathrm{~min}$ in the darkness. Cells were pelleted and resuspended in $500 \mu \mathrm{l}$ staining medium containing $1 \mu \mathrm{g} / \mathrm{ml}$ DAPI for the exclusion of dead cells and subjected to the FCM evaluation or FACS sorting. The evaluation was done on the Calibur flow cytometer (Becton Dickinson, USA) and analyzed by FlowJo software (FlowJo LLC, USA). Sorting was done using MA900 cell sorter (Sony, Japan). Sorted live Trf-positive cells were imaged with Nikon Eclipse fluorescent microscope and with ZEISS LSM900 confocal microscope.

\section{Phagocytosis essay}

Cells and cell-free CF from three sea urchins were used in the phagocytosis assay. The cells were washed two times with cold staining medium (3.3X PBS with $20 \mathrm{mM}$ HEPES, $2 \%$ fetal calf serum and $0.09 \%$ Sodium Azid-NaN3, pH7.4) and pelleted at $500 \mathrm{~g}$ and $4^{\circ} \mathrm{C}$. Washed cells of each animal were divided into three aliquots and kept in the staining medium on ice until use. For detection of phagocytosed bacteria, we used either FITC-stained or mCherry expressing E. coli bacteria. Bacteria were diluted to a concentration of $10^{7}$ per $\mathrm{ml}$ and divided into three groups (A, B and C). Group A and C were incubated for 40 min with sea urchin hemolymph at $16^{\circ} \mathrm{C}$ in the dark. Groups B bacteria remained untreated. Group $\mathrm{C}$ bacteria was additionally incubated with antiSpTrf polyclonal antibodies mixture in 1:100 ration in $100 \mu \mathrm{l}$ for $30 \mathrm{~min}$ in the dark. All bacteria samples (A, B, C) were then mixed with the corresponding coelomocyte aliquots of each of three sea urchins and incubated in the dark for $70 \mathrm{~min}$. After the incubation, cells were shortly pelleted and loaded to the hemocytometer for the visual evaluation of phagocytosis using fluorescent microscope Nikon Eclipse with standard fluorescent lamp and company filters, Olympus (model IX81, with company filters). Phagocytosis events were blindly counted (without knowing the type of treatment), using 16 middle squares of the hemocytometer $(n=3)$ for $A$, B and C phagocytosis treatments. Differences in the number of phagocyted cells in different treatments were assessed by Anova two-factor and one-factor analyses.

\section{Acknowledgments:}

The authors are grateful to Prof. L. Courtney Smith from the Department of Biological Sciences in The George Washington University for the anti-SpTrf antibodies, to Dr. Galia Luboshits from the Department of Chemical Engineering in Ariel University for technical assistance with flow cytometry, to Dr. Alon Savidor and Dr. Amir Pri-Or from the de Botton Institute for Protein Profiling of the Weizmann Institute of Science for MS analysis and to Ms. Helena Tuchinsky for technical assistance. Authors also express special gratitude to the Inkscape open-source vector graphics program creators and contributors; the program was used for the creation and refinement of the most figures presented in this article https://sourceforge.net/projects/inkscape/. This work was supported by the Ariel University new faculty seed money. The work of BR was supported by Israel Science Foundation (ISF) grants numbers: $1416 / 19$ and 2841/19. 


\section{Supplementary material}

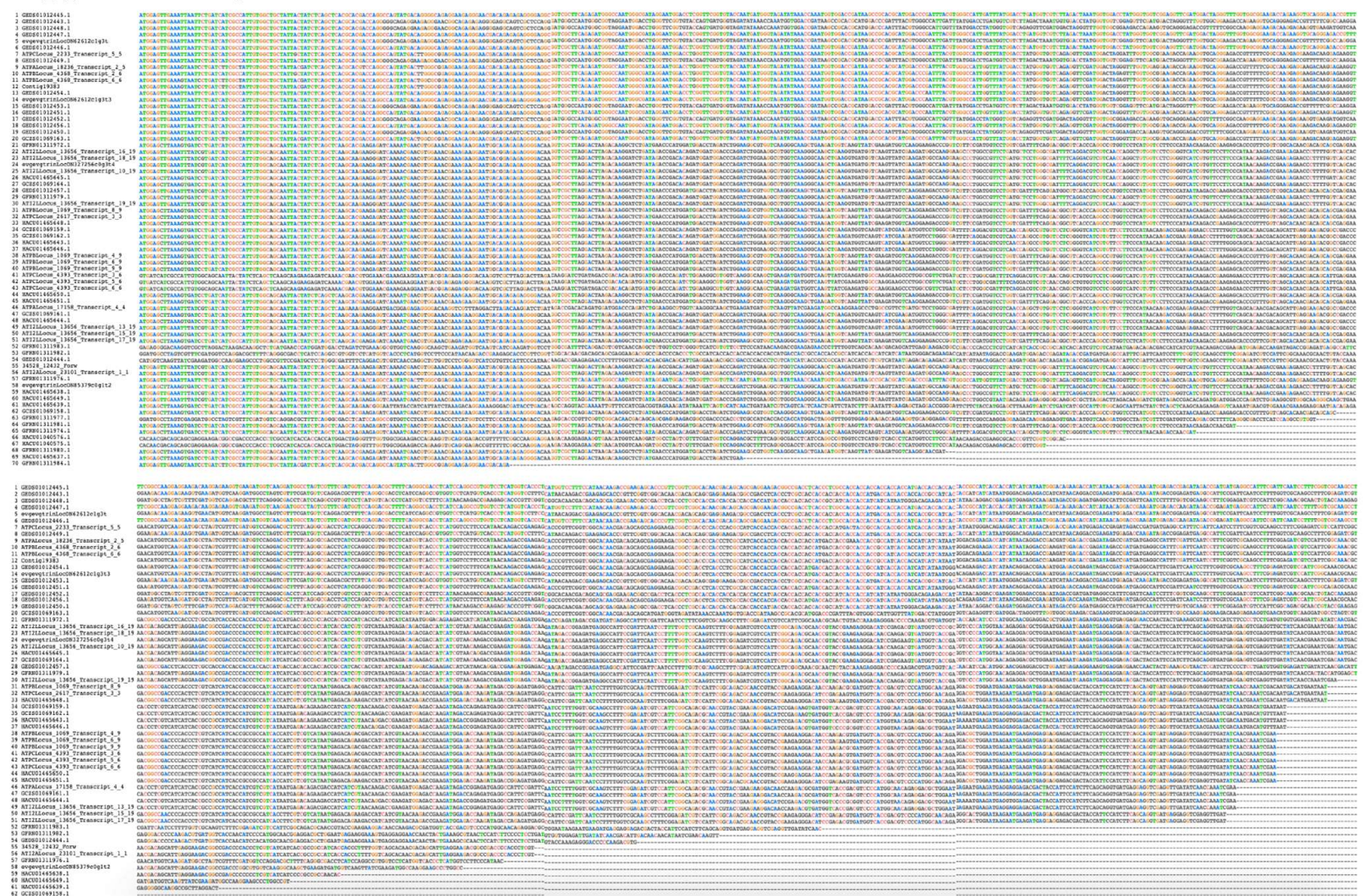

Fig. S1 Unique Trf sequences obtained from publicly available databases.

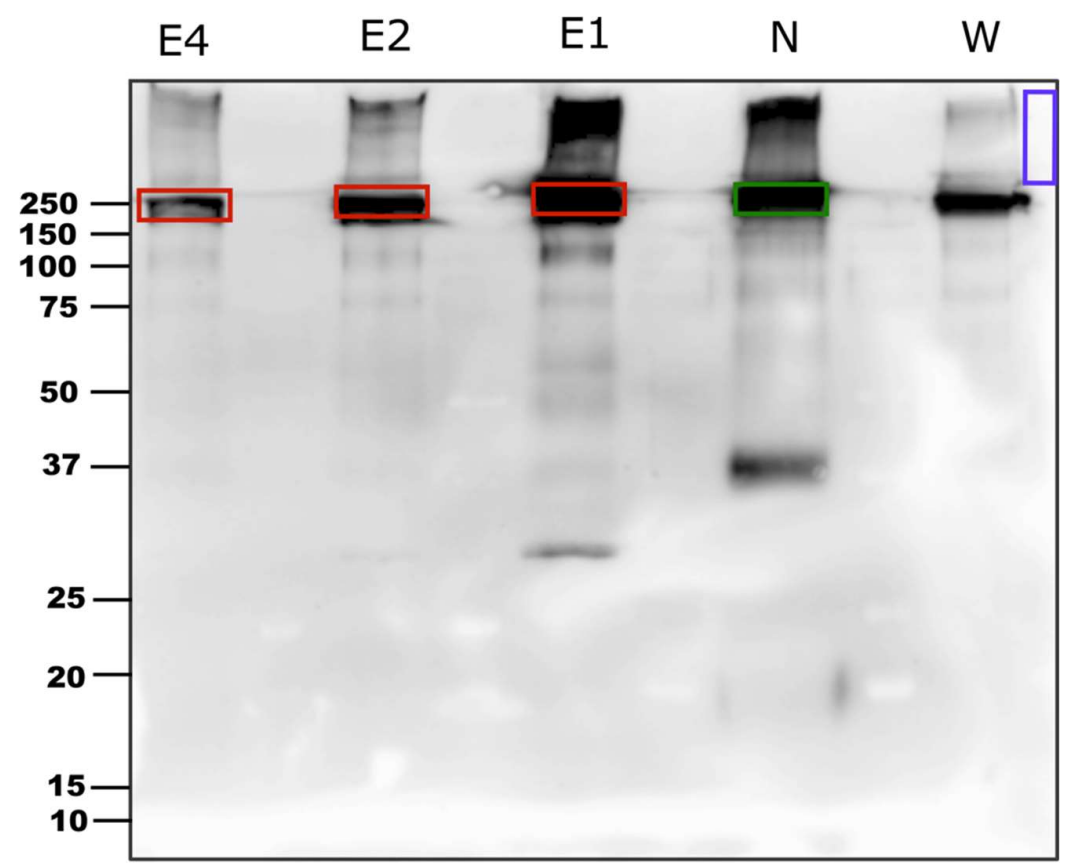

Fig. S2 Western blot of the gel that was used for the mass spectrometry. E1, E2, E4 - elution fractions, N untreated native whole coelomic fluid protein, $\mathrm{W}$ - wash fraction. Inside boxes are the bands that were cut from PAGE gel. In red - elution fractions, in green - native protein from the same animal, in blue - the background control that was cut from the side of the gel. 

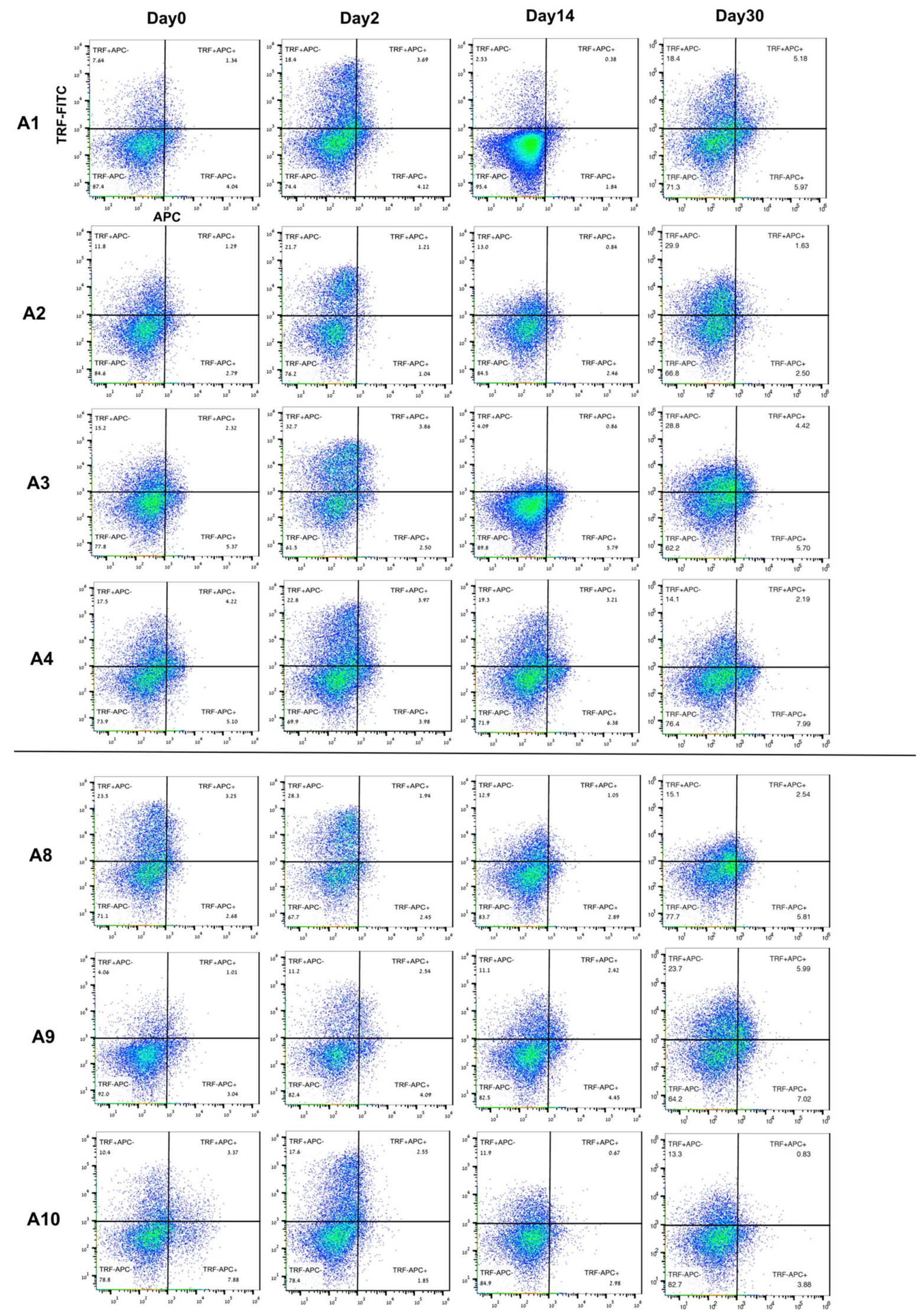

Fig. S3 Trf-positive cell population change over time after injection of either $V$. penaeicida or Acf. A1-A4 - animals challenged with heat-killed $V$. penaeicida, A8-A10 - control animals injected with sterile aCF. Y-axis Trf labeled with AF488, X-axis autofluorescence in APC channel indicates red spherule cells. 
Table S1 accessions of the Trf sequences used to build the phylogenetic tree.

\begin{tabular}{|c|c|c|c|c|c|}
\hline \multicolumn{2}{|c|}{ H. erythrogramma* } & \multicolumn{2}{|c|}{ S. purpuratus*s } & \multicolumn{2}{|l|}{ P. lividus** } \\
\hline JX245053.1 & HE001 & KJ408453.1 & SP001 & HACU01465650.1 & PL001 \\
\hline JQ780274.1 & HE002 & KJ408451.1 & SP002 & HACU01465648.1 & PL002 \\
\hline JX245045.1 & HE003 & KJ408450.1 & SP003 & HACU01465646.1 & PL003 \\
\hline JQ780273.1 & HE004 & KJ408449.1 & SP004 & HACU01465645.1 & PL004 \\
\hline JX245024.1 & HE005 & EF607775.1 & SP005 & HACU01465644.1 & PL005 \\
\hline JX245021.1 & HE006 & EF607759.1 & SP006 & HACU01465643.1 & PL006 \\
\hline JQ780304.1 & HE007 & EF607755.1 & SP007 & GFRN01311981.1 & PL007 \\
\hline JX245020.1 & HE008 & EF607753.1 & SP008 & GFRN01311979.1 & PL008 \\
\hline JQ780312.1 & HE009 & EF607742.1 & SP009 & GFRN01311974.1 & PL009 \\
\hline JQ780311.1 & HE010 & EF607719.1 & SP010 & GFRN01311972.1 & PL010 \\
\hline JQ780305.1 & HE011 & EF607717.1 & SP011 & GEDS01012457.1 & PL011 \\
\hline JQ780303.1 & HE012 & EF607713.1 & SP012 & GEDS01012456.1 & PL012 \\
\hline JQ780287.1 & HE013 & EF066272.1 & SP013 & GEDS01012454.1 & PL013 \\
\hline JQ780284.1 & HE014 & EF066267.1 & SP014 & GEDS01012453.1 & PL014 \\
\hline JQ780276.1 & HE015 & EF066259.1 & SP015 & GEDS01012452.1 & PL015 \\
\hline JQ780275.1 & HE016 & EF066253.1 & SP016 & GEDS01012451.1 & PL016 \\
\hline JQ780271.1 & HE017 & EF066219.1 & SP017 & GEDS01012450.1 & PL017 \\
\hline JQ780270.1 & HE018 & EF066159.1 & SP018 & GEDS01012448.1 & PL018 \\
\hline JQ780269.1 & HE019 & EF065743.1 & SP019 & GEDS01012447.1 & PL019 \\
\hline JQ780268.1 & HE020 & EF065742.1 & SP020 & GEDS01012446.1 & PL020 \\
\hline JQ780266.1 & HE021 & EF065733.1 & SP021 & GEDS01012445.1 & PL021 \\
\hline JQ780265.1 & HE022 & DQ183180.1 & SP022 & GEDS01012443.1 & PL022 \\
\hline JQ780262.1 & HE023 & DQ183182.1 & SP023 & GCZS01069164.1 & PL023 \\
\hline JQ780244.1 & HE024 & DQ183168.1 & SP024 & GCZS01069162.1 & PL024 \\
\hline JQ780233.1 & HE025 & DQ183167.1 & SP025 & GCZS01069159.1 & PL025 \\
\hline
\end{tabular}

* P. lividus, S. purpuratus and H. erythrogramma Trf sequence accession numbers from NCBI protein database **P. lividus sequences from TSA NCBI databases (HACU01, GCZS01, GEDS01, GFRN01)
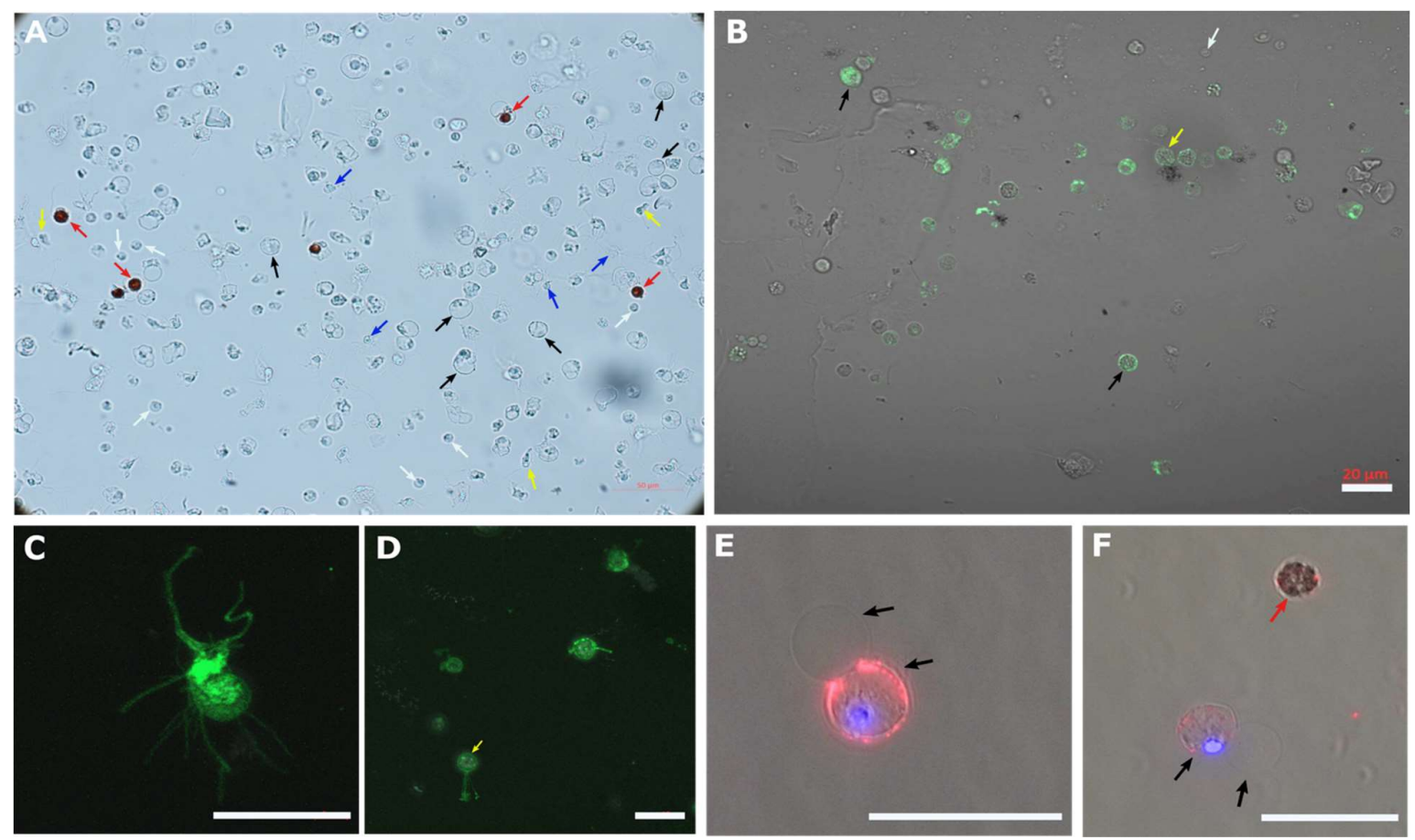

Fig S4. P. lividus coelomocyte types and sorted Trf-positive coelomocytes. A. P. lividus unsorted coelomocytes in whole CF shown in a bright field. B. Sorted Trf-positive cells - petaloid phagocytes and a vibratile cell labeling is clearly seen, other coelomocyte types have a weaker signal. C. Trf protein expression on 
the cell surface and filopodia of a filopodial phagocyte (confocal microscopy) D. Trf-positive $P$. lividus vibratile cell (yellow arrow) (confocal microscopy). $\mathbf{E}$ and F. Membranal interactions of Trf-positive and Trf-negative petaloid phagocytes (black arrows). Scale bars $=20 \mu \mathrm{m}$. Blue arrows - filopodial phagocytes. Red arrows- red spherule cells. Yellow arrows - vibratile cells. Green fluorophore $-2^{\text {nd }} \mathrm{Ab}$ AlexaFluor 488 . Red fluorophore $-2^{\text {nd }}$ $\mathrm{Ab}$ AlexaFluor 567. Blue is DAPI.

\section{References}

1. Smith, L Courtney, Jonathan P Rast, Virginia Brockton, David P Terwilliger, Sham V Nair, Katherine M Buckley, and Audrey J Majeske. "The Sea Urchin Immune System." Invertebrate Survival Journal 3, no. 1 (2006): 25-39.

2. Rast, Jonathan P, L Courtney Smith, Mariano Loza-Coll, Taku Hibino, and Gary W Litman. "Genomic Insights into the Immune System of the Sea Urchin." Science 314, no. 5801 (2006): 952-56.

3. Smith, L Courtney, Vincenzo Arizza, Megan A Barela Hudgell, Gianpaolo Barone, Andrea G Bodnar, Katherine M Buckley, Vincenzo Cunsolo, Nolwenn M Dheilly, Nicola Franchi, Sebastian D Fugmann, Ryohei Furukawa, Jose Garcia- Arraras, John H. Henson, Taku Hibino, Zoe H. Irons, Cheng Man Lun Chun Li, Audrey J. Majeske, Matan Oren, Patrizia Pagliara, Annalisa Pinsino, David A. Raftos, Jonathan P. Rast, Bakary Samasa, Domenico Schillaci, Catherine S. Schrankel, Loredana Stabili, Klara Stensväg, and Elisse Sutton. "Echinodermata: The Complex Immune System in Echinoderms." In Advances in Comparative Immunology, 409-501: Springer, 2018.

4. Nair, Sham V, Heather Del Valle, Paul S Gross, David P Terwilliger, and L Courtney Smith. "Macroarray Analysis of Coelomocyte Gene Expression in Response to Lps in the Sea Urchin. Identification of Unexpected Immune Diversity in an Invertebrate." Physiological genomics 22, no. 1 (2005): 33-47.

5. Roth, Mattias O, Adam G Wilkins, Georgina M Cooke, David A Raftos, and Sham V Nair. "Characterization of the Highly Variable Immune Response Gene Family, He185/333, in the Sea Urchin, Heliocidaris Erythrogramma." PLoS ONE 9, no. 10 (2014): e62079.

6. Sherman, Lauren S, Catherine S Schrankel, Kristy J Brown, and L Courtney Smith. "Extraordinary Diversity of Immune Response Proteins among Sea Urchins: NickelIsolated Sp185/333 Proteins Show Broad Variations in Size and Charge." PLoS ONE 10, no. 9 (2015): e0138892.

7. Majeske, Audrey J, Matan Oren, Sandro Sacchi, and L Courtney Smith. "Single Sea Urchin Phagocytes Express Messages of a Single Sequence from the Diverse Sp185/333 Gene Family in Response to Bacterial Challenge." Journal of Immunology 193, no. 11 (2014): 5678-88.

8. Terwilliger, David P, Katherine M Buckley, Virginia Brockton, Nicole J Ritter, and L Courtney Smith. "Distinctive Expression Patterns of 185/333 Genes in the Purple Sea Urchin, Strongylocentrotus Purpuratus: An Unexpectedly Diverse Family of Transcripts in Response to Lps, B-1, 3-Glucan, and Dsrna." BMC Molecular Biology 8, no. 1 (2007): 16.

9. Terwilliger, David P, Katherine M Buckley, Dhruti Mehta, Priya G Moorjani, and L Courtney Smith. "Unexpected Diversity Displayed in Cdnas Expressed by the Immune Cells of the Purple Sea Urchin, Strongylocentrotus Purpuratus." Physiological Genomics 26, no. 2 (2006): 134-44.

10. Dheilly, Nolwenn M, Sham V Nair, L Courtney Smith, and David A Raftos. "Highly Variable Immune-Response Proteins (185/333) from the Sea Urchin, Strongylocentrotus Purpuratus: Proteomic Analysis Identifies Diversity within and between Individuals." Journal of Immunology 182, no. 4 (2009): 2203-12.

11. Majeske, Audrey J, Taras K Oleksyk, and L Courtney Smith. "The Sp185/333 Immune Response Genes and Proteins Are Expressed in Cells Dispersed within All Major Organs of the Adult Purple Sea Urchin." Innate immunity 19, no. 6 (2013): 569-87. 
12. Oren, Matan, Benyamin Rosental, Teresa S Hawley, Gi-Young Kim, Jacob Agronin, Caroline R Reynolds, Leon Grayfer, and L Courtney Smith. "Individual Sea Urchin Coelomocytes Undergo Somatic Immune Gene Diversification." Frontiers in immunology 10 (2019): 1298.

13. Brockton, Virginia, John H Henson, David A Raftos, Audrey J Majeske, Young-Ok Kim, and L Courtney Smith. "Localization and Diversity of 185/333 Proteins from the Purple Sea Urchin-Unexpected Protein-Size Range and Protein Expression in a New Coelomocyte Type." Journal of Cell Science 121, no. 3 (2008): 339-48.

14. Lun, Cheng Man, Catherine S Schrankel, Hung-Yen Chou, Sandro Sacchi, and L Courtney Smith. "A Recombinant Sp185/333 Protein from the Purple Sea Urchin Has Multitasking Binding Activities Towards Certain Microbes and Pamps." Immunobiology 221, no. 8 (2016): 889-903.

15. Chou, Hung-Yen, Cheng Man Lun, and L Courtney Smith. "Sptransformer Proteins from the Purple Sea Urchin Opsonize Bacteria, Augment Phagocytosis, and Retard Bacterial Growth." PloS one 13, no. 5 (2018): e0196890.

16. Lun, Cheng Man, Barney M Bishop, and L Courtney Smith. "Multitasking Immune Sp185/333 Protein, Rsptransformer-E1, and Its Recombinant Fragments Undergo Secondary Structural Transformation Upon Binding Targets." Journal of Immunology 198, no. 7 (2017): 2957-66.

17. Buckley, Katherine M, and L Courtney Smith. "Extraordinary Diversity among Members of the Large Gene Family, 185/333, from the Purple Sea Urchin, Strongylocentrotus Purpuratus." BMC Molecular Biology 8, no. 1 (2007): 68.

18. Buckley, Katherine M, David P Terwilliger, and L Courtney Smith. "Sequence Variations in 185/333 Messages from the Purple Sea Urchin Suggest Posttranscriptional Modifications to Increase Immune Diversity." The Journal of Immunology 181, no. 12 (2008): 8585-94.

19. Oren, Matan, Megan A Barela Hudgell, Brian D’Allura, Jacob Agronin, Alexandra Gross, Daniele Podini, and L Courtney Smith. "Short Tandem Repeats, Segmental Duplications, Gene Deletion, and Genomic Instability in a Rapidly Diversified Immune Gene Family." BMC genomics 17, no. 1 (2016): 900.

20. Oren, Matan, Megan A Barela Hudgell, Preethi Golconda, Cheng Lun Man, and L Courtney Smith. "Genomic Instability and Shared Mechanisms for Gene Diversification in Two Distant Immune Gene Families: The Echinoid 185/333 and the Plant Nbs-Lrr." In Evolution of Immune Systems, edited by Davide Malagoli, 295-307: London, UK: Elsevier-Academic Press, 2016.

21. Smith, L Courtney, and Cheng Man Lun. "The Sptransformer Gene Family (Formerly Sp185/333) in the Purple Sea Urchin and the Functional Diversity of the Anti-Pathogen Rsptransformer-E1 Protein." Frontiers in immunology 8 (2017): 725.

22. Lun, Cheng Man, Robin L Samuel, Susan D Gillmor, Anthony Boyd, and L Courtney Smith. "The Recombinant Sea Urchin Immune Effector Protein, Rsptransformer-E1, Binds to Phosphatidic Acid and Deforms Membranes." Frontiers in Immunology 8 (2017): 481.

23. Boudouresque, Charles F, and Marc Verlaque. "Paracentrotus Lividus." In Developments in Aquaculture and Fisheries Science, 297-327: Elsevier, 2013.

24. Unuma, T. "Gonadal Growth and Its Relationship to Aquaculture in Sea Urchins." The sea urchin: from basic biology to aquaculture (2002): 115-27.

25. Gildor, Tsvia, Assaf Malik, Noa Sher, Linor Avraham, and Smadar Ben-Tabou de-Leon. "Quantitative Developmental Transcriptomes of the Mediterranean Sea Urchin Paracentrotus Lividus." Marine genomics 25 (2016): 89-94.

26. Pinsino, Annalisa, and Valeria Matranga. "Sea Urchin Immune Cells as Sentinels of Environmental Stress." Developmental \& Comparative Immunology 49, no. 1 (2015): 198205.

27. Ghosh, Julie, Katherine M Buckley, Sham V Nair, David A Raftos, Chase Miller, Audrey J Majeske, Taku Hibino, Jonathan P Rast, Mattias Roth, and L Courtney Smith. "Sp185/333: A 
Novel Family of Genes and Proteins Involved in the Purple Sea Urchin Immune Response." Developmental \& Comparative Immunology 34, no. 3 (2010): 235-45.

28. Hasegawa, Masami, Hirohisa Kishino, and Taka-aki Yano. "Dating of the Human-Ape Splitting by a Molecular Clock of Mitochondrial DNA." Journal of molecular evolution 22, no. 2 (1985): 160-74.

29. Kumar, Sudhir, Glen Stecher, Michael Li, Christina Knyaz, and Koichiro Tamura. "Mega X: Molecular Evolutionary Genetics Analysis across Computing Platforms." Molecular biology and evolution 35, no. 6 (2018): 1547-49.

30. Smith, L Courtney, Teresa S Hawley, John H Henson, Audrey J Majeske, Matan Oren, and Benyamin Rosental. "Methods for Collection, Handling, and Analysis of Sea Urchin Coelomocytes." Methods in cell biology 150 (2019): 357-89.

31. Hira, Jonathan, Deanna Wolfson, Aaron John Christian Andersen, Tor Haug, and Klara Stensvåg. "Autofluorescence Mediated Red Spherulocyte Sorting Provides Insights into the Source of Spinochromes in Sea Urchins." Scientific reports 10, no. 1 (2020): 1-9.

32. Becker, Pierre T, Emilie Egea, and Igor Eeckhaut. "Characterization of the Bacterial Communities Associated with the Bald Sea Urchin Disease of the Echinoid Paracentrotus Lividus." Journal of Invertebrate Pathology 98, no. 2 (2008): 136-47.

33. Rast, JP, Z Pancer, and EH Davidson. "New Approaches Towards an Understanding of Deuterostome Immunity." Origin and evolution of the vertebrate immune system (2000): 316.

34. Matranga, Virginia, Annalisa Pinsino, M Celi, A Natoli, R Bonaventura, HC Schröder, and WEG Müller. "Monitoring Chemical and Physical Stress Using Sea Urchin Immune Cells." Echinodermata (2005): 85-110.

35. Ghosh, Julie, Cheng Man Lun, Audrey J Majeske, Sandro Sacchi, Catherine S Schrankel, and L Courtney Smith. "Invertebrate Immune Diversity." Developmental \& Comparative Immunology 35, no. 9 (2011): 959-74.

36. Kurtz, Joachim. "Specific Memory within Innate Immune Systems." Trends in immunology 26, no. 4 (2005): 186-92.

37. Letunic, Ivica, and Peer Bork. "Interactive Tree of Life (Itol) V4: Recent Updates and New Developments." Nucleic acids research 47, no. W1 (2019): W256-W59.

38. Smith, L Courtney, Roy J Britten, and Eric H Davidson. "Lipopolysaccharide Activates the Sea Urchin Immune System." Developmental and Comparative Immunology 19, no. 3 (1995): 217-24. 